Supporting Information for:

\title{
Planar Chiral Cyclic Ether: Asymmetric Resolution and Chirality Transformation
}

Katsuhiko Tomooka,* Nobuyuki Komine, Daisuke Fujiki, Takeshi Nakai, and Syun-ichi Yanagitsuru

Department of Applied Chemistry, Graduate School of Science and Engineering, Tokyo Institute of Technology, Meguro-ku, Tokyo 152-8552, Japan

\section{General Experimental.}

All reactions were carried out with dry glassware under an atmosphere of argon otherwise noted. Dry THF, $\mathrm{CH}_{2} \mathrm{Cl}_{2}$, benzene and hexane were purchased from Kanto Chemical Co., Inc. ${ }^{1} \mathrm{H}$ NMR spectra were recorded on a Varian Gemine-2000 (300 MHz) or a Varian Mercury $(300 \mathrm{MHz})$ using $\mathrm{CDCl}_{3}$ or $\left[\mathrm{D}_{6}\right]$ benzene as solvent: $\mathrm{CHCl}_{3}\left({ }^{1} \mathrm{H}, \delta\right.$ 7.26) or benzene $\left({ }^{1} \mathrm{H}, \delta 7.15\right)$ were used as internal references. Peak multiplicities were given as followed: s, singlet; $d$, doublet; $t$, triplet; q, quartet; m, multiplet; br, broad. ${ }^{13} \mathrm{C}$ NMR spectra were recorded on a Varian Gemine-2000 $(75 \mathrm{MHz})$ or a Varian Mercury $(75 \mathrm{MHz})$ using $\mathrm{CDCl}_{3}$ or $\left[\mathrm{D}_{6}\right]$ benzene as solvent: $\mathrm{CDCl}_{3}$ $\left({ }^{13} \mathrm{C}, \delta 77.1\right)$ or $\mathrm{C}_{6} \mathrm{D}_{6}\left({ }^{13} \mathrm{C}, \delta 128.0\right)$ were used as internal references. Optical rotations were measured on a digital polarimeter (JASCO DIP-370). Infrared spectra were recorded on a Fourier transfer infrared spectrophotometer (Perkin Elmer SpectrumOne) as neat liquid on $\mathrm{NaCl}$ plates. Analytical high performance liquid chromatography (HPLC) was performed on a SHIMADZU SPD-6A equipped with a SHIMADZU LC-9A or a JASCO CD-2095 equipped with JASCO PU-2089 using Daicel CHIRALCEL OD, OD-H column $(0.46 \mathrm{~cm}$ x $25 \mathrm{~cm})$, Daicel CHIRALPAK AD column $(0.46 \mathrm{~cm}$ x $25 \mathrm{~cm})$. Thin-layer chromatography (TLC) was carried out on silica gel 60 $\mathrm{F}_{254}$ (Merk 5715) plates. Developed plates were visualized by ultraviolet illumination at $254 \mathrm{~nm}$ and by heating on a hot plate after staining with a $4 \%$ solution of phosphomolybdic acid in ethanol, a $2.5 \%$ solution of $p$-anisaldehyde in ethanol, or an aqueous solution of potassium permanganate. Column chromatography was performed using Merck Kieselgel 60, Fuji Silysia FL100D (neutral) and Kanto 60N (63 $210 \mathrm{~nm}$ ). Elemental analyses were performed by the Analytical Facility at the Research Laboratory of Resources Utilization, Tokyo Institute of Technology.

\section{Preparation of (3E,7Z)-3,7-Dimethyl-2,5,6,9-tetrahydro-oxonine (1a)}
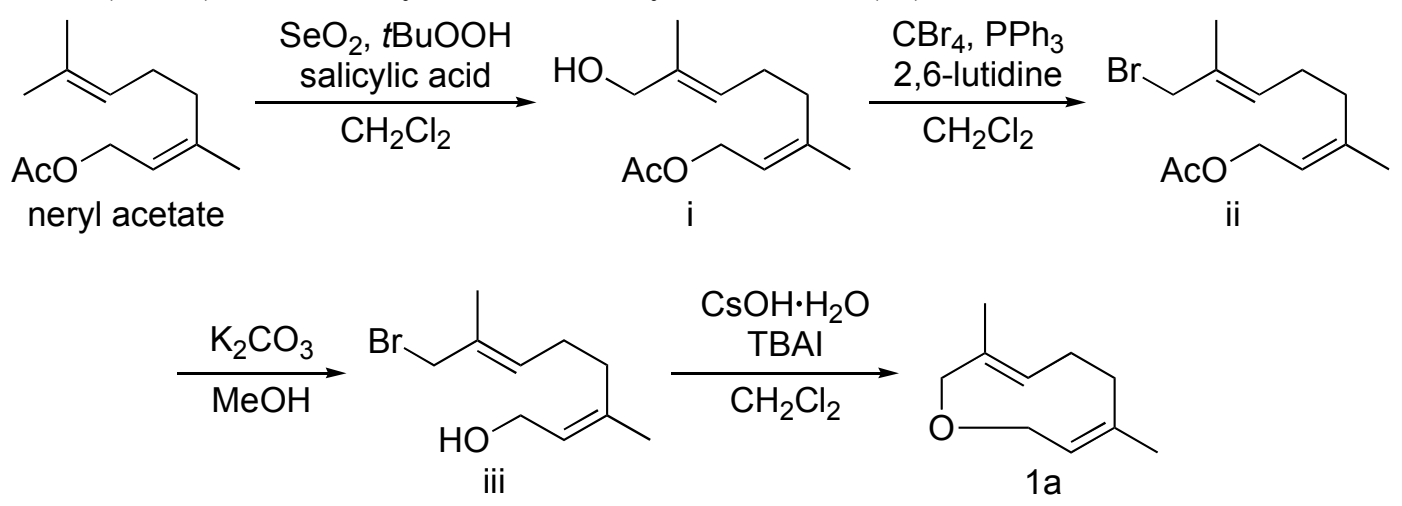

Cyclic ether 1a was prepared by Marshall's procedure (J. A. Marshall, J. Lebreton, J. Org. Chem. 1988, 53, 4108-4112) with a slight modification. 


\section{Acetic acid (2Z,6E)-8-hydroxy-3,7-dimethyl-octa-2,6-dienyl ester (i)}

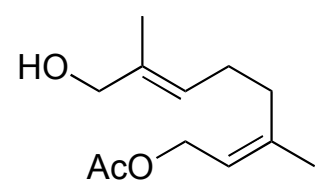

To a solution of catalytic amount of $\mathrm{SeO}_{2}(79.6 \mathrm{mg}, 0.695 \mathrm{mmol})$ and salicylic acid $(96.0 \mathrm{mg}, 0.695 \mathrm{mmol})$ in dry $\mathrm{CH}_{2} \mathrm{Cl}_{2}(10 \mathrm{~mL})$ at room temperature was added anhydrous TBHP in $\mathrm{CH}_{2} \mathrm{Cl}_{2}(30 \mathrm{~mL})$, which was prepared from TBHP in water $70 \mathrm{wt} \%(3.5 \mathrm{~mL}, 25.0 \mathrm{mmol})$ by azeotropic distillation of water and $\mathrm{CH}_{2} \mathrm{Cl}_{2}$. And neryl acetate $(1.5 \mathrm{~mL}, 6.95 \mathrm{mmol})$ was added to the reaction mixture, then the resulting mixture was stirred at that temperature for $23 \mathrm{~h}$. The reaction was quenched with aq satd $\mathrm{Na}_{2} \mathrm{~S}_{2} \mathrm{O}_{3}$ and extracted with $\mathrm{CH}_{2} \mathrm{Cl}_{2}$. The combined organic phase was dried over $\mathrm{Na}_{2} \mathrm{SO}_{4}$, filtered and the solvent was removed in vacuo. The residue was purified by silica gel chromatography, affording $233 \mathrm{mg}(17 \%)$ of recovered starting material (hexane/AcOEt = 10/1), $224 \mathrm{mg}$ $(15 \%)$ of aldehyde (hexane/AcOEt $=6 / 1)$ and $861 \mathrm{mg}(58 \%)$ of alcohol $\mathbf{i}$ (hexane/AcOEt $=3 / 1$ to $1 / 1) .{ }^{1} \mathrm{H}$ NMR (300 MHz, CDCl $)$ ): $\delta 5.39$ (brs, 1H), 5.36 (t, $J=7.2 \mathrm{~Hz}, 1 \mathrm{H}), 4.56$ (d, $J=7.2 \mathrm{~Hz}, 2 \mathrm{H}), 3.99$ (s, 2H), 2.16 (s, 2H), $2.15(\mathrm{~s}, 2 \mathrm{H}), 2.05$ (s, 3H), 1.77 (s, 3H), $1.66(\mathrm{~s}, 3 \mathrm{H}) ;{ }^{13} \mathrm{C} \mathrm{NMR}\left(75 \mathrm{MHz}, \mathrm{CDCl}_{3}\right): \delta 171.2,142.0,135.7,125.0$, 119.7, 68.9, 61.3, 31.8, 26.0, 23.5, 21.2, 13.8; IR (neat, $\mathrm{cm}^{-1}$ ): 3432, 2937, 2862, 1739, 1670, 1445, 1379, 1236, $1022,954,852,743$.

\section{Acetic acid (2Z,6E)-8-bromo-3,7-dimethyl-octa-2,6-dienyl ester (ii)}

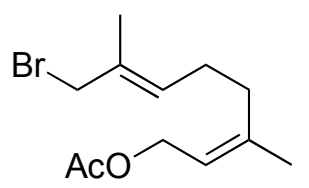

To a solution of $\mathbf{i}(88.2 \mathrm{mg}, 0.412 \mathrm{mmol})$ in dry $\mathrm{CH}_{2} \mathrm{Cl}_{2}(40 \mathrm{~mL})$ at $0{ }^{\circ} \mathrm{C}$ was added 2,6-lutidine $(0.048 \mathrm{~mL}$, $0.412 \mathrm{mmol}$ ) and tetrabromomethane $(276 \mathrm{mg}, 0.831 \mathrm{mmol})$. After the resulting mixture was cooled to $0{ }^{\circ} \mathrm{C}$, triphenylphosphine $(229 \mathrm{mg}, 0.873 \mathrm{mmol}$ ) was added to the solution. The reaction mixture was stirred at that temperature for $50 \mathrm{~min}$, concentrated in vacuo and the residue was purified by silica gel chromatography (hexane/AcOEt $=1 / 0$ to 6/1) to afford $106 \mathrm{mg}$ of acetate ii $(93 \%) .{ }^{1} \mathrm{H} \mathrm{NMR}\left(300 \mathrm{MHz}, \mathrm{CDCl}_{3}\right)$ : $\delta 5.56(\mathrm{br}, 1 \mathrm{H})$, $5.36(\mathrm{t}, J=7.5 \mathrm{~Hz}, 1 \mathrm{H}), 4.53(\mathrm{~d}, J=7.5 \mathrm{~Hz}, 2 \mathrm{H}), 3.93(\mathrm{~s}, 2 \mathrm{H}), 2.14(\mathrm{~s}, 2 \mathrm{H}), 2.13(\mathrm{~s}, 3 \mathrm{H}), 2.03$ (s, $3 \mathrm{H}), 1.75$ (s, $6 \mathrm{H}) ;{ }^{13} \mathrm{C}$ NMR (75 MHz, $\mathrm{CDCl}_{3}$ ): $\delta 170.8,141.6,132.7,130.0,119.7,61.0,41.4,31.4,26.9,23.5,21.2,14.8$; IR $\left(\right.$ neat, $\left.\mathrm{cm}^{-1}\right): 3455,2938,1738,1664,1442,1379,1365,1234,1144,1023,956,609$.

\section{(2Z,6E)-8-Bromo-3,7-dimethyl-octa-2,6-dien-1-ol (iii)}

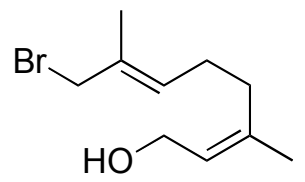

A slurry of acetate ii $(98.4 \mathrm{mg}, 0.378 \mathrm{mmol})$ and catalytic amount of $\mathrm{K}_{2} \mathrm{CO}_{3}$ in methanol $(4 \mathrm{~mL})$ at $0{ }^{\circ} \mathrm{C}$ was stirred for $2 \mathrm{~h}$. The mixture was diluted with water and extracted with $\mathrm{Et}_{2} \mathrm{O}$. The combined organic phase was dried over $\mathrm{Na}_{2} \mathrm{SO}_{4}$, filtered and the solvent was removed in vacuo to afford $72.2 \mathrm{mg}$ of a volatile oil iii (87\%). 
This allyl bromide was used in next reaction without further purification. ${ }^{1} \mathrm{H}$ NMR $\left(300 \mathrm{MHz}, \mathrm{CDCl}_{3}\right): \delta 5.57$ (brs, 1H), 5.43 (t, $J=6.9 \mathrm{~Hz}, 1 \mathrm{H}), 4.10$ (d, $J=6.9 \mathrm{~Hz}, 2 \mathrm{H}), 3.95(\mathrm{~s}, 2 \mathrm{H}), 2.14(\mathrm{~s}, 2 \mathrm{H}), 2.13(\mathrm{~s}, 2 \mathrm{H}), 1.75(\mathrm{~s}, 6 \mathrm{H}), 1.56$ (s, $1 \mathrm{H}) ;{ }^{13} \mathrm{C}$ NMR (75 MHz, $\mathrm{CDCl}_{3}$ ): $\delta 138.8,132.7,130.3,124.9,59.1,41.6,31.2,26.8,23.5,14.8$; IR (neat, $\mathrm{cm}^{-1}$ ): $3341,2930,2859,1666,1445,1377,1206,1142,1001,904,841,609$.

\section{(3E,7Z)-3,7-Dimethyl-2,5,6,9-tetrahydro-oxonine (1a)}

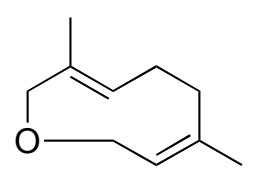

To a solution of crude iii $(54.7 \mathrm{mg}, 0.235 \mathrm{mmol})$ in dry $\mathrm{CH}_{2} \mathrm{Cl}_{2}(2.3 \mathrm{~mL})$ was added TBAI $(8.7 \mathrm{mg}, 0.0235$ $\mathrm{mmol})$ and cesium hydroxide monohydrate $(197 \mathrm{mg}, 1.18 \mathrm{mmol})$ at $0^{\circ} \mathrm{C}$. The reaction mixture was vigorously stirred at that temperature for $24 \mathrm{~h}$. The reaction was quenched by diluting with water and was extracted with $\mathrm{CH}_{2} \mathrm{Cl}_{2}$. The combined organic phase was dried over $\mathrm{Na}_{2} \mathrm{SO}_{4}$, filtered and the solvent was removed in vacuo. The residue was purified by silica gel chromatography (hexane/AcOEt $=1 / 0$ to 20/1) to afford $16.1 \mathrm{mg}$ of cyclic ether 1a $(45 \%)$ as a volatile colorless oil : bp. $48-50{ }^{\circ} \mathrm{C} .{ }^{1} \mathrm{H}$ NMR $\left(300 \mathrm{MHz}, \mathrm{CDCl}_{3}\right): \delta 5.54(\mathrm{~m}, 1 \mathrm{H}), 5.36(\mathrm{dd}, J=$ 11.7, $4.8 \mathrm{~Hz}, 1 \mathrm{H}), 4.18(\mathrm{~d}, J=9.6 \mathrm{~Hz}, 1 \mathrm{H}), 3.86(\mathrm{dd}, J=12.9,4.8 \mathrm{~Hz}, 1 \mathrm{H}), 3.73(\mathrm{~d}, J=9.6 \mathrm{~Hz}, 1 \mathrm{H}), 3.59(\mathrm{dd}, J=$ 12.9, $11.7 \mathrm{~Hz}, 1 \mathrm{H}), 2.09-1.98(\mathrm{~m}, 4 \mathrm{H}), 1.75$ (s, 3H), $1.52(\mathrm{~s}, 3 \mathrm{H}) ;{ }^{13} \mathrm{C}$ NMR $\left(75 \mathrm{MHz}, \mathrm{CDCl}_{3}\right): \delta 137.5,134.0$, 133.3, 128.5, 78.8, 62.4, 33.5, 25.7, 25.6, 16.6; IR (neat, $\mathrm{cm}^{-1}$ ): 2932, 2865, 2727, 2671, 1655, 1456, 1376, 1344, 1276, 1233, 1204, 1174, 1137, 1116, 1045, 1011, 955, 932, 893, 871, 823, 791, 741; Analytical HPLC (column: CHIRALCEL OD-H $(0.46 \mathrm{~cm} \times 25 \mathrm{~cm})$, eluent: hexane $/ \mathrm{PrOH}=500 / 1$, flow rate: $0.3 \mathrm{~mL} / \mathrm{min}$, detection: UV $220 \mathrm{~nm}$, temperature: RT) $t_{R}=12.6 \mathrm{~min}$ for $(S)$-isomer, $14.9 \mathrm{~min}$ for $(R)$-isomer; Optical rotation value: $[\alpha]_{\mathrm{D}}{ }^{29}=$ $+97.6\left(c=0.85, \mathrm{CHCl}_{3}\right)$ for $>98 \%$ ee $(R),[\alpha]_{\mathrm{D}}^{25}=-25.3\left(c=2.60, \mathrm{CHCl}_{3}\right)$ for $26 \%$ ee $(S)$.

\section{(3E,7Z)-2,5,6,9-Tetrahydro-oxonine (1b)}
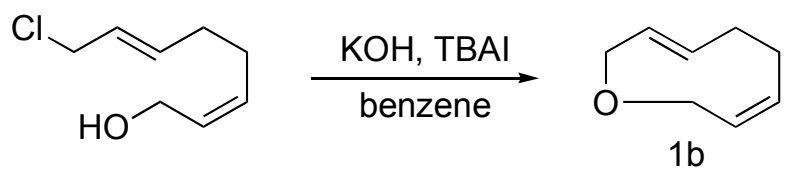

A suspension of $1.00 \mathrm{~g}$ of TBAI and $1.00 \mathrm{~g}$ of powdered $\mathrm{KOH}$ in dry benzene $(40 \mathrm{~mL})$ was stirred at room temperature and then a solution of (2Z,6E)-8-chloro-octa-2,6-dien-1-ol (100mg, $0.622 \mathrm{mmol})$ in dry benzene (20 $\mathrm{mL}$ ) was added to the mixture very slowly. After $12 \mathrm{~h}$ the resulting suspension was filtrated through a pad of celite and concentrated in vacuo to give a yellow oil, which was purified by silica gel chromatography, affording $25 \mathrm{mg}$ of a volatile oil $\mathbf{1 b}(33 \%)$. ${ }^{1} \mathrm{H}$ NMR $\left(300 \mathrm{MHz}, \mathrm{CDCl}_{3}\right): \delta 5.71$ (dddd, $\left.J=11.7,4.8,1.5,1.5 \mathrm{~Hz}, 1 \mathrm{H}\right), 5.56$ (ddd, $J=15.3,7.5,1.5 \mathrm{~Hz}, 1 \mathrm{H}), 5.40(\mathrm{dd}, J=10.5,3.9 \mathrm{~Hz}, 1 \mathrm{H}), 5.30(\mathrm{~m}, 1 \mathrm{H}), 4.38(\mathrm{dd}, J=9.9,3.9 \mathrm{~Hz}, 1 \mathrm{H}), 3.81$ $(\mathrm{dd}, J=12.9,4.8 \mathrm{~Hz}, 1 \mathrm{H}), 3.74(\mathrm{dd}, J=10.5,9.9 \mathrm{~Hz}, 1 \mathrm{H}), 3.42(\mathrm{dd}, J=12.9,11.7 \mathrm{~Hz}, 1 \mathrm{H}), 2.44(\mathrm{~m}, 1 \mathrm{H}), 2.31(\mathrm{~m}$, 1H), $1.86(\mathrm{~m}, 1 \mathrm{H}), 1.51(\mathrm{~m}, 1 \mathrm{H})$; Analytical HPLC (column: CHIRALPAK AD (0.46 cm x $25 \mathrm{~cm})$, eluent: hexane, flow rate: $0.3 \mathrm{~mL} / \mathrm{min}$, detection: UV $210 \mathrm{~nm}$, temperature: $\left.0{ }^{\circ} \mathrm{C}\right) t_{R}=104.8 \mathrm{~min}$ for $(R)$-isomer, 116.2 $\min$ for $(S)$-isomer; Optical rotation value: $[\alpha]_{\mathrm{D}}{ }^{25}=-6.60\left(c=0.45, \mathrm{CHCl}_{3}\right)$ for $72 \%$ ee $(S)$. 


\section{General Procedure for Asymmetric [2,3]-Wittig Rearrangement in the Presence of $(S, S)$-Bis(dihydrooxazol)}

(2)

To a solution of $(S, S)$-bis(dihydrooxazol) 2 (1.5 equiv) in hexane at $-78{ }^{\circ} \mathrm{C}$ was added $t \mathrm{BuLi}$ (1.5 equiv). The reaction mixture was stirred for $15 \mathrm{~min}$ at $-78{ }^{\circ} \mathrm{C}$ and a solution of cyclic ether $\mathbf{1}$ in hexane was added to the mixture. The reaction mixture was stirred at $-78^{\circ} \mathrm{C}$ for $3 \mathrm{~h}$. The reaction was quenched with $\mathrm{H}_{2} \mathrm{O}$ and the aqueous layer was extracted with $\mathrm{Et}_{2} \mathrm{O}$. The combined extracts were washed twice with brine, dried over anhydrous $\mathrm{MgSO}_{4}$, filtered, and concentrated in vacuo to afford the corresponding alcohol 3 (3a: $20 \%,>98 \%$ dr, $94 \%$ ee $(R, R)$; 3b: 66\%, $>98 \% \mathrm{dr}, 15 \%$ ee $(R, R))$ and recovered cyclic ether $\mathbf{1}(\mathbf{1 a}: 72 \%, 26 \%$ ee $(S) ; 1 \mathbf{b}: 16 \%, 72 \%$ ee $(S))$. The enantio-purity of alcohol $\mathbf{3}$ was determined by ${ }^{1} \mathrm{H}$ NMR of MTPA ester.

$(S, S)$-Bis(dihydrooxazol) (2)

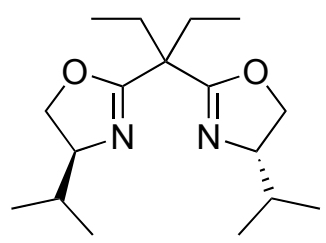

$(S, S)$-Bis(dihydrooxazol) 2 was prepared by the reported method: S. E. Denmark, N. Nakajima, O. J.-C. Nicaise, A. M. Faucher, J. P. Edwards, J. Org. Chem. 1995, 60, 4884.

(1R,6R)-6-Isopropenyl-3-methyl-cyclohex-2-enol (3a)

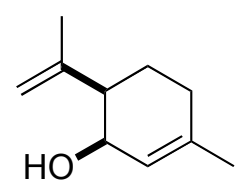

${ }^{1} \mathrm{H}$ NMR $\left(300 \mathrm{MHz}, \mathrm{CDCl}_{3}\right)$ : $\delta 5.67$ (m, 1H), 4.99 (s, 1H), 4.81 (s, 1H), 4.12 (brs, 1H), 2.17-2.04 (m, 3H), 1.83 (s, $3 \mathrm{H}), 1.80-1.74(\mathrm{~m}, 1 \mathrm{H}), 1.72(\mathrm{~s}, 3 \mathrm{H}), 1.65-1.55(\mathrm{~m}, 1 \mathrm{H}), 1.48$ (brs, $1 \mathrm{H}) ;{ }^{13} \mathrm{C}$ NMR $\left(75 \mathrm{MHz}, \mathrm{CDCl}_{3}\right): \delta 146.7$, 139.9, 122.5, 111.8, 63.9, 46.2, 31.3, 23.5, 22.7, 21.0; IR (neat, $\mathrm{cm}^{-1}$ ): 3352, 3088, 2936, 1676, 1649, 1450, 1379, $1245,1210,1176,1156,1114,1069,1025,957,915,886,849,816,723,681$.

\section{$(R)$-MTPA ester of $(1 R, 6 R)-3 a$}

(S)- $\alpha$-Methoxy- $\alpha$-(trifluoromethyl)phenylacetyl chloride ((S)-MTPA-Cl) was prepared from the corresponding acid to the procedure of Mosher: J. A. Dale, D. L. Dull, H. S. Mosher, J. Org. Chem. 1969, 34, 2543.

${ }^{1} \mathrm{H}$ NMR $\left(300 \mathrm{MHz}, \mathrm{CDCl}_{3}\right): \delta 7.50(\mathrm{~m}, 2 \mathrm{H}), 7.36(\mathrm{~m}, 3 \mathrm{H}), 5.73(\mathrm{~m}, 1 \mathrm{H}), 5.53(\mathrm{~m}, 1 \mathrm{H}), 4.89(\mathrm{~d}, J=1.2 \mathrm{~Hz}, 1 \mathrm{H})$, $4.75(\mathrm{~s}, 1 \mathrm{H}), 3.50(\mathrm{~d}, J=1.2 \mathrm{~Hz}, 3 \mathrm{H}), 2.3-2.0(\mathrm{~m}, 5 \mathrm{H}), 1.77(\mathrm{~s}, 3 \mathrm{H}), 1.72(\mathrm{~s}, 3 \mathrm{H})$.

\section{$(R)$-MTPA ester of $(1 S, 6 S)$-3a}

${ }^{1} \mathrm{H}$ NMR $\left(300 \mathrm{MHz}, \mathrm{CDCl}_{3}\right): \delta 7.50(\mathrm{~m}, 2 \mathrm{H}), 7.36(\mathrm{~m}, 3 \mathrm{H}), 5.73(\mathrm{~m}, 1 \mathrm{H}), 5.53(\mathrm{~m}, 1 \mathrm{H}), 4.66(\mathrm{~d}, J=1.2 \mathrm{~Hz}, 1 \mathrm{H})$, 4.59 (s, 1H), 3.52 (d, $J=1.2 \mathrm{~Hz}, 3 \mathrm{H}), 2.3-2.0(\mathrm{~m}, 5 \mathrm{H}), 1.77$ (s, 3H), $1.72(\mathrm{~s}, 3 \mathrm{H})$. 


\section{(R)-6-Isopropenyl-3-methyl-cyclohex-2-enone [(-)-isopiperitenone]}<smiles>C=C(C)[C@H]1CCC(C)=C[C@H]1O</smiles>

$3 a$

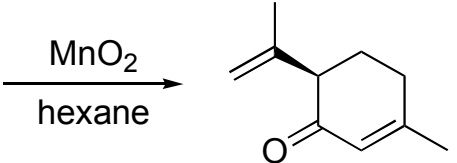

西

To a solution of $(1 R, 6 R)$-6-Isopropenyl-3-methyl-cyclohex-2-enol 3a $(20.0 \mathrm{mg}, 0.131 \mathrm{mmol}, 53 \%$ ee $)$ in dry hexane $(5 \mathrm{~mL})$ was added $\mathrm{MnO}_{2}(186.4 \mathrm{mg}, 2.63 \mathrm{mmol})$ at room temperature. The mixture was stirred vigorously at room temperature for $12 \mathrm{~h}$ and filtrated through a pad of Celite. After removal of the solvent, the residue was purified by silica gel chromatography to afford $16.1 \mathrm{mg}$ of ketone $(82 \%)$ as a volatile oil. ${ }^{1} \mathrm{H}$ NMR (300 MHz, $\left.\mathrm{CDCl}_{3}\right): \delta 5.89(\mathrm{~d}, J=1.5 \mathrm{~Hz}, 1 \mathrm{H}), 4.94(\mathrm{~d}, J=1.5 \mathrm{~Hz}, 1 \mathrm{H}), 4.75(\mathrm{~d}, J=1.5 \mathrm{~Hz}, 1 \mathrm{H}), 2.94(\mathrm{dd}, J=10.5,5.4 \mathrm{~Hz}$, 1H), $2.32(\mathrm{~m}, 2 \mathrm{H}), 2.03(\mathrm{~m}, 2 \mathrm{H}), 1.94(\mathrm{~s}, 3 \mathrm{H}), 1.74(\mathrm{~d}, J=1.5 \mathrm{~Hz}, 3 \mathrm{H}) ;{ }^{13} \mathrm{C}$ NMR $\left(75 \mathrm{MHz}, \mathrm{CDCl}_{3}\right): \delta 199.5$, 162.0, 143.4, 126.8, 113.6, 53.9, 30.4, 27.7, 24.3, 20.7; IR (neat, $\mathrm{cm}^{-1}$ ): 3306, 2862, 2728, 2364, 2334, 1669, 1543, 1448, 1381, 1270, 1236, 1205, 1160, 1139, 1098, 1064, 1042, 1009, 982, 951, 913, 888, 849, 806, 762, 727, 677, 640; Optical rotation value: $[\alpha]_{\mathrm{D}}{ }^{31}=-25.0(c=0.81, \mathrm{EtOH})$ for $53 \%$ ee; lit: $[\alpha]_{\mathrm{D}}=+63.4(c=1.35$, EtOH $)$ for (S)-isomer according to the literature: A. R. Pinder, T. A. Anglea, Tetrahedron. 1987, 43, 5537.

\section{$(1 R, 6 R)-6-I s o p r o p e n y l-c y c l o h e x-2-e n-1-o l ~(3 b)$}

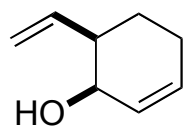

${ }^{1} \mathrm{H}$ NMR (300 MHz, $\left.\mathrm{CDCl}_{3}\right): \delta 6.04-5.80(\mathrm{~m}, 3 \mathrm{H}), 5.21(\mathrm{~d}, J=1.2 \mathrm{~Hz}, 1 \mathrm{H}), 5.17$ (ddd, $\left.J=5.4,1.8,1.2 \mathrm{~Hz}, 1 \mathrm{H}\right)$, 4.12 (brs, 1H), 2.38 (m, 1H), 2.26-1.98 (m, 2H), 1.80 (s, 3H), $1.68(\mathrm{~m}, 2 \mathrm{H}) ;{ }^{13} \mathrm{C}$ NMR $\left(75 \mathrm{MHz}, \mathrm{CDCl}_{3}\right) \delta$ 139.1, $130.9,128.7,116.5,66.4,43.6,24.8,22.2$;

\section{(Z)-3,7-Dimethyl-3,4-epoxy-2,5,6,9-tetrahydro-oxonine (4)}

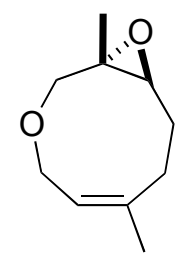

A soluton of dimethyldioxiran $(5.2 \mathrm{~mL}$ of $0.07 \mathrm{M}$ in acetone, $0.361 \mathrm{mmol})$, which was dried over molecular sieves $(4 \AA)$, was added at $0{ }^{\circ} \mathrm{C}$ to a stirred solution of cyclic ether $1 \mathbf{a}(50.0 \mathrm{mg}, 0.328 \mathrm{mmol})$ in $\mathrm{dry} \mathrm{CH}_{2} \mathrm{Cl}_{2}(10$ $\mathrm{mL}$ ). After stirring for $30 \mathrm{~min}$ at that temperature, the solvent was removed in vacuo. The residue was purified by silica gel chromatography (FL100D, pentane $/ \mathrm{Et}_{2} \mathrm{O}=10: 1$ ) to afford $41.0 \mathrm{mg}$ of epoxide $2(91 \%)$ as a single diastereomer. ${ }^{1} \mathrm{H}$ NMR $\left(300 \mathrm{MHz}, \mathrm{C}_{6} \mathrm{D}_{6}\right)$ : $\delta 5.38$ (ddd, $\left.J=11.6,5.0,1.4 \mathrm{~Hz}, 1 \mathrm{H}\right), 4.05(\mathrm{~d}, J=10.7 \mathrm{~Hz}, 1 \mathrm{H}), 3.91$ $(\mathrm{dd}, J=13.0,5.0 \mathrm{~Hz}, 1 \mathrm{H}), 3.62(\mathrm{dd}, J=13.0,11.6 \mathrm{~Hz}, 1 \mathrm{H}), 2.94(\mathrm{~d}, J=10.7 \mathrm{~Hz}, 1 \mathrm{H}), 2.65(\mathrm{dd}, J=11.4,3.0 \mathrm{~Hz}$, $1 \mathrm{H}), 1.96(\mathrm{t}, J=13.5 \mathrm{~Hz}, 1 \mathrm{H}), 1.65-1.56(\mathrm{~m}, 1 \mathrm{H}), 1.48-1.44(\mathrm{~m}, 1 \mathrm{H}), 1.43(\mathrm{~d}, J=1.4 \mathrm{~Hz}, 3 \mathrm{H}), 1.16(\mathrm{~s}, 3 \mathrm{H})$, 
0.95-0.81 (m, 1H); ${ }^{13} \mathrm{C}$ NMR (75 MHz, $\left.\mathrm{C}_{6} \mathrm{D}_{6}\right): \delta$ 134.7, 126.7, 77.3, 66.3, 64.8, 57.2, 28.0, 25.4, 22.8, 16.9; IR (neat, $\mathrm{cm}^{-1}$ ): 2931, 2872, 2730, 2689, 1958, 1922, 1895, 1862, 1727, 1663, 1464, 1383, 1341, 1265, 1223, 1191, 1167, 1139, 1065, 1030, 1002, 978, 932, 911, 896, 869, 832, 784, 722, 603; Analytical HPLC (column: CHIRALCEL OD $(0.46 \mathrm{~cm} \times 25 \mathrm{~cm})$, eluent: hexane $/ \mathrm{PrOH}=300 / 1$, flow rate: $0.5 \mathrm{~mL} / \mathrm{min}$, detection: UV 210 $\mathrm{nm}$, temperature: $\mathrm{RT}) t_{R}=30.6 \mathrm{~min}$ for $(3 R, 4 R)$-isomer, $33.7 \mathrm{~min}$ for $(3 S, 4 S)$-isomer.

\section{(E)-3,7-dimethyl-7,8-epoxy-2,5,6,9-tetrahydro-oxonine (5)}

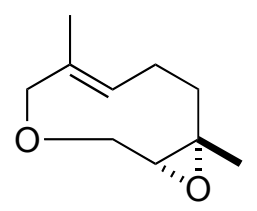

${ }^{1} \mathrm{H}$ NMR $\left(300 \mathrm{MHz}, \mathrm{CDCl}_{3}\right): \delta 5.04(\mathrm{~m}, 1 \mathrm{H}), 4.08(\mathrm{~d}, J=9.9 \mathrm{~Hz}, 1 \mathrm{H}), 4.03(\mathrm{dd}, J=13.2,2.4 \mathrm{~Hz}, 1 \mathrm{H}), 3.43(\mathrm{~d}, J=$ $9.9 \mathrm{~Hz}, 1 \mathrm{H}), 2.82(\mathrm{dd}, J=13.2,9.9 \mathrm{~Hz}, 1 \mathrm{H}), 2.64(\mathrm{dd}, J=9.9,2.4 \mathrm{~Hz}, 1 \mathrm{H}), 1.97(\mathrm{~m}, 1 \mathrm{H}), 1.71(\mathrm{~m}, 2 \mathrm{H}), 1.48(\mathrm{~s}$, $3 \mathrm{H}), 1.07$ (s, 3H), $1.00(\mathrm{~m}, 1 \mathrm{H}) ;{ }^{13} \mathrm{C}$ NMR $\left(75 \mathrm{MHz}, \mathrm{C}_{6} \mathrm{D}_{6}\right): \delta$ 132.1, 131.6, 78.1, 65.1, 64.1, 61.2, 35.0, 25.5, 22.8, 15.4 .

\section{3,7-dimethyl-3,4,7,8-diepoxy-2,5,6,9-tetrahydro-oxonine (6)}

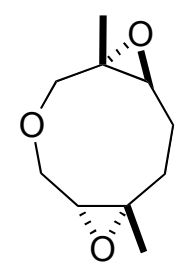

${ }^{1} \mathrm{H}$ NMR $\left(300 \mathrm{MHz}, \mathrm{CDCl}_{3}\right) \delta 4.14(\mathrm{dd}, J=13.4,2.4 \mathrm{~Hz}, 1 \mathrm{H}), 4.09(\mathrm{~d}, J=10.8 \mathrm{~Hz}, 1 \mathrm{H}), 3.30(\mathrm{dd}, J=13.4,10.3$ Hz, 1H), 2.99 (d, $J=10.8 \mathrm{~Hz}, 1 \mathrm{H}), 2.93$ (dd, $J=10.3,2.4 \mathrm{~Hz}, 1 \mathrm{H}), 2.88(\mathrm{~m}, 1 \mathrm{H}), 2.20(\mathrm{~m}, 2 \mathrm{H}), 1.46(\mathrm{~m}, 2 \mathrm{H}), 1.36$ (s, 6H); ${ }^{13} \mathrm{C} \mathrm{NMR}\left(75 \mathrm{MHz}, \mathrm{CDCl}_{3}\right): \delta 77.3,66.0,65.6,64.3,62.1,56.8,30.7,23.7,22.9,15.6$.

\section{(Z)-3,7-Dimethyl-2,3,4,5,6,9-hexahydro-oxonin-4-ol (7)}

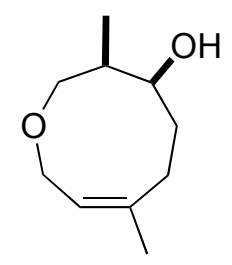

To a stirred solution of 9-BBN (1.57 $\mathrm{mL}$ of $0.5 \mathrm{M}$ 9-BBN in THF, $0.787 \mathrm{mmol})$ and dry THF $(1 \mathrm{~mL})$ was added cyclic ether $1 \mathrm{a}(50.0 \mathrm{mg}, 0.328 \mathrm{mmol})$ in dry THF $(5 \mathrm{~mL})$ at room temperature. After heating under reflux for $5 \mathrm{~h}$, the organoborane was oxidized by adding $0.5 \mathrm{~mL}$ of $\mathrm{MeOH}, 0.5 \mathrm{~mL}$ of $6 \mathrm{~N} \mathrm{NaOH}$ and $0.5 \mathrm{~mL}$ of aq $30 \% \mathrm{H}_{2} \mathrm{O}_{2}$ successively. The mixture was heated at $40{ }^{\circ} \mathrm{C}$ for $1 \mathrm{~h}$ to ensure complete oxidaiton, then cooled to room temperature. The reaction was quenched with aq satd $\mathrm{NaHCO}_{3}$ and the aqueous layer was extracted with $\mathrm{Et}_{2} \mathrm{O}$. 
The combined extracts were washed twice with brine, dried over anhydrous $\mathrm{MgSO}_{4}$. After removal of the solvent, the residue was purified by silica gel chromatography to afford $50.8 \mathrm{mg}$ of alcohol 7 (91\%) as a volatile oil. ${ }^{1} \mathrm{H}$ NMR (300 MHz, $\left.\mathrm{CDCl}_{3}\right): \delta 5.30(\mathrm{t}, J=4.2 \mathrm{~Hz}, 1 \mathrm{H}), 4.22(\mathrm{dq}, J=15.0,1.8 \mathrm{~Hz}, 1 \mathrm{H}), 3.86(\mathrm{dd}, J=15.0,2.4 \mathrm{~Hz}$, $1 \mathrm{H}), 3.76(\mathrm{~m}, 1 \mathrm{H}), 3.65(\mathrm{dd}, J=12.0,7.2 \mathrm{~Hz}, 1 \mathrm{H}), 3.42(\mathrm{dd}, J=12.0,3.6 \mathrm{~Hz}, 1 \mathrm{H}), 2.89(\mathrm{~m}, 1 \mathrm{H}), 1.97(\mathrm{~m}, 1 \mathrm{H})$, $1.86(\mathrm{dt}, J=18.0,4.8 \mathrm{~Hz}, 1 \mathrm{H}), 1.78(\mathrm{~m}, 2 \mathrm{H}), 1.74(\mathrm{~s}, 3 \mathrm{H}), 0.92(\mathrm{~d}, J=7.2 \mathrm{~Hz}, 3 \mathrm{H}) ;{ }^{13} \mathrm{C} \mathrm{NMR}\left(75 \mathrm{MHz}, \mathrm{CDCl}_{3}\right)$ : $\delta$ 141.8, 122.1, 74.7, 73.1, 68.1, 40.3, 30.7, 28.2, 23.1, 12.1; IR (neat, $\mathrm{cm}^{-1}$ ): 3338, 2926, 2364, 1669, 1450, 1381, 1270, 1160, 1139, 1100, 1064, 1042, 1009, 982, 951, 913, 888, 849, 806, 762; Anal. Calcd for $\mathrm{C}_{10} \mathrm{H}_{18} \mathrm{O}_{2}$ : C, 70.55; H, 10.66. Found: C, 70.16; H, 10.29.

\section{Enantioselective Epoxidation of 1a}

Following the literature procedure: M. Palucki, G. J. McCormic, E. N. Jacobsen, Tetrahedron Lett. 1995, 36, 5457.

$(R, R)-8(10.5 \mathrm{mg}, 16.5 \mu \mathrm{mol})$ and NMO $(193 \mathrm{mg}, 1.65 \mathrm{mmol})$ were added at $-78{ }^{\circ} \mathrm{C}$ to a solution of cyclic ether $1 \mathrm{a}(50.0 \mathrm{mg}, 0.328 \mathrm{mmol})$ in dry $\mathrm{CH}_{2} \mathrm{Cl}_{2}(5 \mathrm{~mL})$. To the mixture, $m$ CPBA (114 mg, $\left.0.656 \mathrm{mmol}\right)$ was added at $-78^{\circ} \mathrm{C}$. After stirring for $45 \mathrm{~min}$ at $-78^{\circ} \mathrm{C}$, the reaction was quenched with dimethylsulfide and aq $\mathrm{NaOH}(2 \mathrm{~N})$. The aqueous layer was extracted with $\mathrm{Et}_{2} \mathrm{O}$. The crude mixture was washed with water, dried over $\mathrm{Na}_{2} \mathrm{SO}_{4}$, concentrated in vacuo, and purified by flash chromatography to afford $22.8 \mathrm{mg}$ of epoxide 4 ( $41 \%,>98 \% \mathrm{dr}, 68 \%$ ee $(3 S, 4 S))$ and $11.9 \mathrm{mg}$ of recovered starting material $\mathbf{1 a}(24 \%, 70 \%$ ee $(S))$. The enantio-purity of cyclic ether $\mathbf{1 a}$ and epoxide $\mathbf{4}$ were determined by chiral HPLC analysis.

$(R, R)-8$

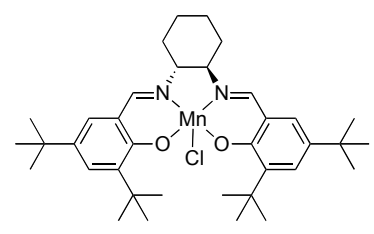

\section{Enantioselective Hydroboration Procedure by $(+)-\mathrm{Ipc}_{2} \mathrm{BH}$}

To a stirred suspension of $(+)-\mathrm{Ipc}_{2} \mathrm{BH}(0.393 \mathrm{mmol})$ (prepared by following the literature procedure: $\mathrm{H}$. C. Brown, M. C. Desai, P. K. Jadhav, J. Org. Chem. 1982, 47, 5065.) in dry THF (3 mL) at $-50{ }^{\circ} \mathrm{C}$ was added a solution of cyclic ether $1 \mathrm{a}(50.0 \mathrm{mg}, 0.328 \mathrm{mmol})$ in dry THF $(4 \mathrm{~mL})$. The reaction mixture was allowed to rise to $-10{ }^{\circ} \mathrm{C}$ gradually. The organoborane was immediately oxidized by adding $0.5 \mathrm{~mL}$ of $\mathrm{MeOH}, 0.5 \mathrm{~mL}$ of aq $\mathrm{NaOH}$ $(6 \mathrm{~N})$ and $0.5 \mathrm{~mL}$ of aq $30 \% \mathrm{H}_{2} \mathrm{O}_{2}$ successively. The mixture was stirred at $40{ }^{\circ} \mathrm{C}$ for $1 \mathrm{~h}$. The reaction was quenched with sat. $\mathrm{NaHCO}_{3}$ aq. and the aqueous layer was extracted with $\mathrm{Et}_{2} \mathrm{O}$. The combined extracts were washed with brine and dried over anhydrous $\mathrm{MgSO}_{4}$. After removal of the solvent, the residue was purified by silica gel chromatography to afford $28.0 \mathrm{mg}$ of alcohol $7\left(50 \%, 89 \%\right.$ ee $(3 S, 4 R)$; determined by ${ }^{1} \mathrm{H}$ NMR of 
MTPA ester)) and $21.8 \mathrm{mg}$ of recovered cyclic ether 1a (43\%, >98\% ee $(R)$; determined by chiral HPLC)).

\section{(3S,4R)-(7Z)-3,7-Dimethyl-2,3,4,5,6,9-hexahydro-oxonin-4-ol (R)-MTPA ester}

${ }^{1} \mathrm{H}$ NMR (300 MHz, $\left.\mathrm{CDCl}_{3}\right): \delta 7.51(\mathrm{~m}, 2 \mathrm{H}), 7.40$ (m, 3H), 5.38 (brs, 1H), 5.15 (ddd, $\left.J=9.9,3.9,2.1 \mathrm{~Hz}, 1 \mathrm{H}\right)$, $4.23(\mathrm{~d}, J=15.0 \mathrm{~Hz}, 1 \mathrm{H}), 3.90(\mathrm{dd}, J=15.0,5.7 \mathrm{~Hz}, 1 \mathrm{H}), 3.70(\mathrm{dd}, J=12.0,5.7 \mathrm{~Hz}, 1 \mathrm{H}), 3.65(\mathrm{dd}, J=12.0,2.4$ $\mathrm{Hz}, 1 \mathrm{H}), 3.52$ (d, $J=1.2 \mathrm{~Hz}, 3 \mathrm{H}), 3.40$ (dd, $J=15.0,1.2 \mathrm{~Hz}, 1 \mathrm{H}), 3.03(\mathrm{~m}, 1 \mathrm{H}), 1.99$ (m, 1H), 1.90-1.75 (m, 5H), $1.02(\mathrm{~d}, J=7.5 \mathrm{~Hz}, 3 \mathrm{H})$.

\section{(3R,4S)-(7Z)-3,7-Dimethyl-2,3,4,5,6,9-hexahydro-oxonin-4-ol (R)-MTPA ester}

${ }^{1} \mathrm{H}$ NMR (300 MHz, CDCl$): \delta 7.51(\mathrm{~m}, 2 \mathrm{H}), 7.40(\mathrm{~m}, 3 \mathrm{H}), 5.38$ (brs, $\left.1 \mathrm{H}\right), 5.15$ (ddd, $\left.J=9.9,3.9,2.1 \mathrm{~Hz}, 1 \mathrm{H}\right)$, $4.23(\mathrm{~d}, J=15.0 \mathrm{~Hz}, 1 \mathrm{H}), 3.90(\mathrm{dd}, J=15.0,5.7 \mathrm{~Hz}, 1 \mathrm{H}), 3.70(\mathrm{dd}, J=12.0,5.7 \mathrm{~Hz}, 1 \mathrm{H}), 3.65(\mathrm{dd}, J=12.0,2.4$ $\mathrm{Hz}, 1 \mathrm{H}), 3.52(\mathrm{~d}, J=1.2 \mathrm{~Hz}, 3 \mathrm{H}), 3.40(\mathrm{dd}, J=15.0,1.2 \mathrm{~Hz}, 1 \mathrm{H}), 3.03(\mathrm{~m}, 1 \mathrm{H}), 1.99(\mathrm{~m}, 1 \mathrm{H}), 1.90-1.75(\mathrm{~m}, 5 \mathrm{H})$, $0.96(\mathrm{~d}, J=7.5 \mathrm{~Hz}, 3 \mathrm{H})$.

\section{4-Isopropenyl-3-methyl-3-vinyl-tetrahydro-furan (9)}<smiles>C=C[C@]1(C)COC[C@H]1C(=C)C</smiles>

To a solution of cyclic ether 1a $(100 \mathrm{mg}, 0.6569 \mathrm{mmol})$ at room temperature in dry $\mathrm{CH}_{2} \mathrm{Cl}_{2}(4 \mathrm{~mL})$ was added $\mathrm{PdCl}_{2}(\mathrm{PhCN})_{2}(25.2 \mathrm{mg}, 0.06569 \mathrm{mmol})$. After stirring for $2 \mathrm{~h}$ at that temperature, the mixture was filtrated through Florisil (60-100 mesh). The solvent was removed in vacuo. The crude product was purified by silica gel chromatography to afford $82.0 \mathrm{mg}$ of $9\left(82 \%,>98 \% \mathrm{dr}\right.$ analyzed by $\left.{ }^{1} \mathrm{H} \mathrm{NMR}\right) .{ }^{1} \mathrm{H} \mathrm{NMR}\left(300 \mathrm{MHz}, \mathrm{CDCl}_{3}\right): \delta$ $5.88(\mathrm{dd}, J=18.0,10.5 \mathrm{~Hz}, 1 \mathrm{H}), 5.06(\mathrm{~d}, J=10.5 \mathrm{~Hz}, 1 \mathrm{H}), 5.05(\mathrm{~d}, J=18.0 \mathrm{~Hz}, 1 \mathrm{H}), 4.87(\mathrm{~m}, 1 \mathrm{H}), 4.73(\mathrm{~m}, 1 \mathrm{H})$, $4.08(\mathrm{dd}, J=8.7,7.7 \mathrm{~Hz}, 1 \mathrm{H}), 3.91(\mathrm{dd}, J=8.7,7.7 \mathrm{~Hz}, 1 \mathrm{H}), 3.83(\mathrm{~d}, J=8.4 \mathrm{~Hz}, 1 \mathrm{H}), 3.58(\mathrm{~d}, J=8.4 \mathrm{~Hz}, 1 \mathrm{H})$, $2.56(\mathrm{dd}, J=7.7,7.7 \mathrm{~Hz}, 1 \mathrm{H}), 1.71(\mathrm{~s}, 3 \mathrm{H}), 1.24(\mathrm{~s}, 3 \mathrm{H}) ;{ }^{13} \mathrm{C}$ NMR $\left(75 \mathrm{MHz}, \mathrm{CDCl}_{3}\right): \delta 143.0,141.1,113.1$, 112.9, 78.9, 71.6, 56.8, 47.9, 23.6, 23.2; IR (neat, $\mathrm{cm}^{-1}$ ): 2928, 2858, 2334, 1734, 1640, 1458, 1379, 1263, 1122, $1075,913,893$. 


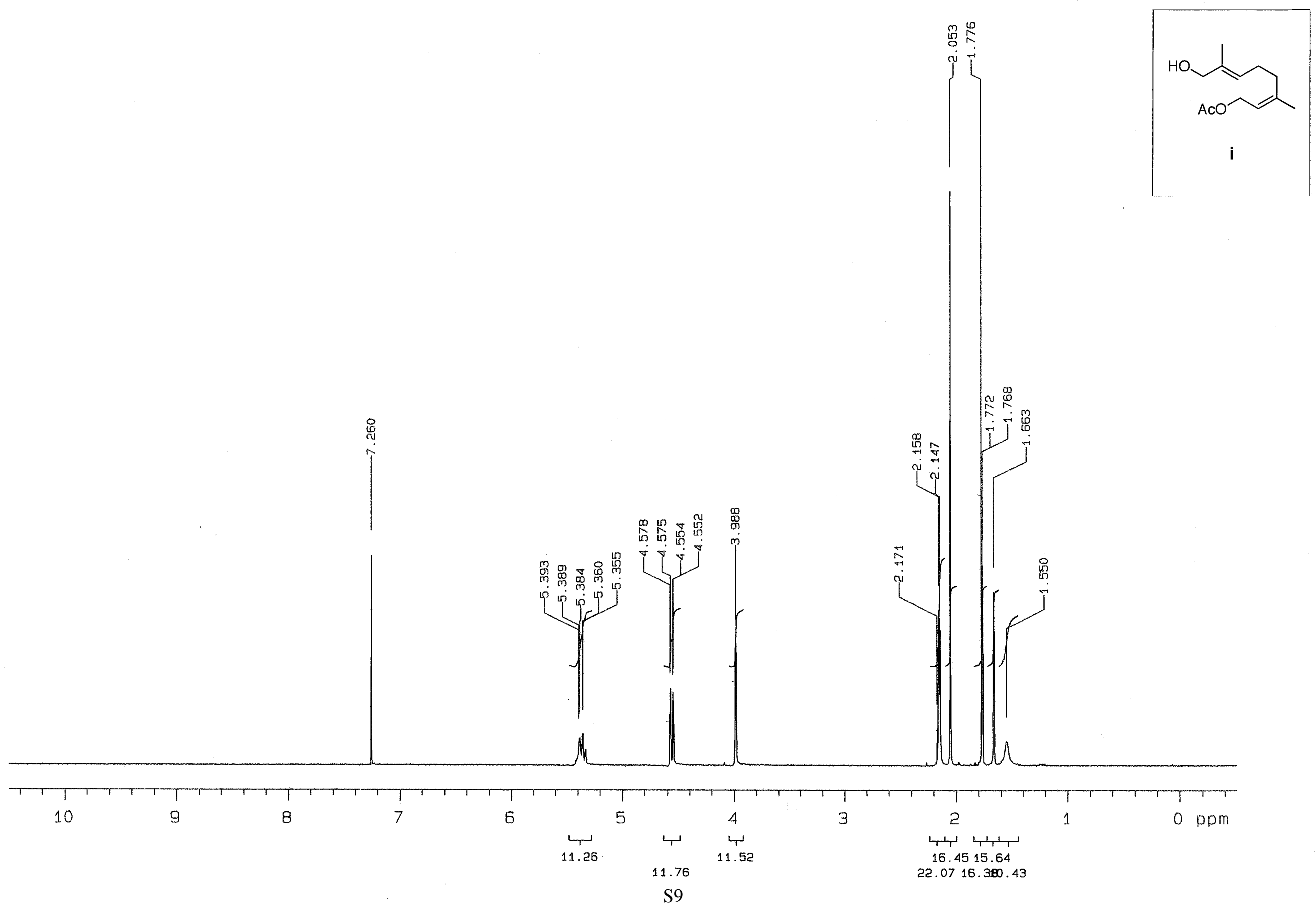



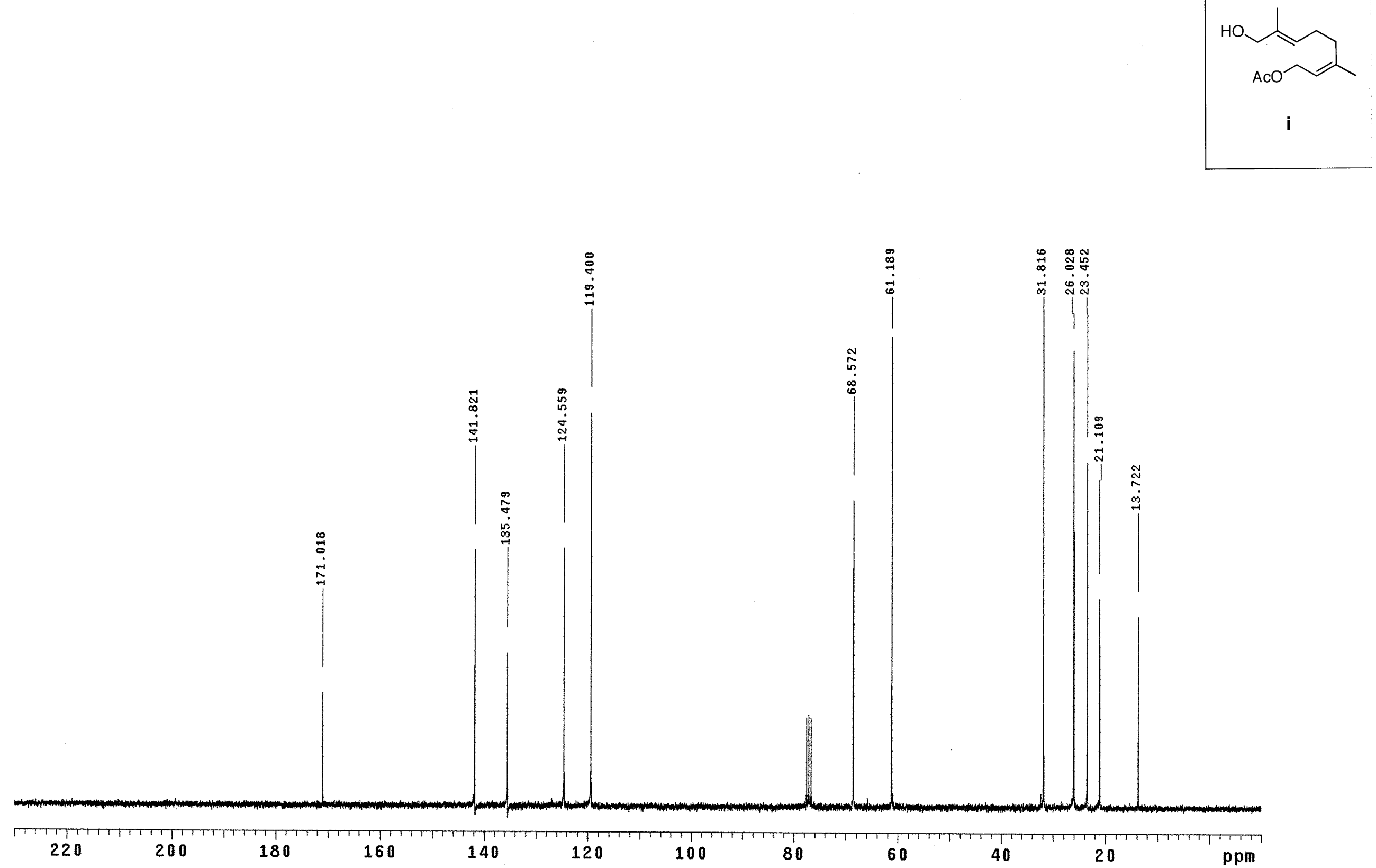

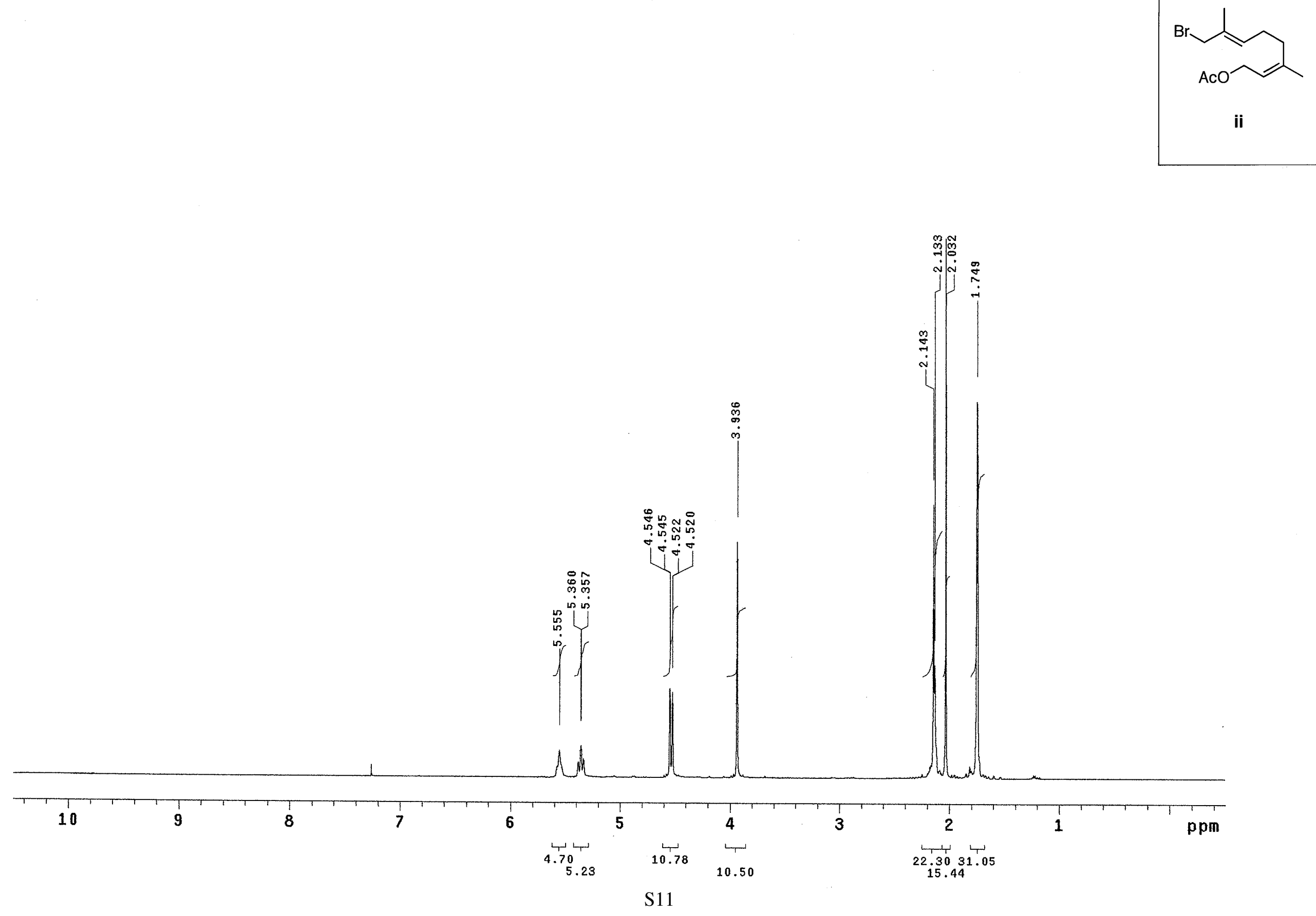

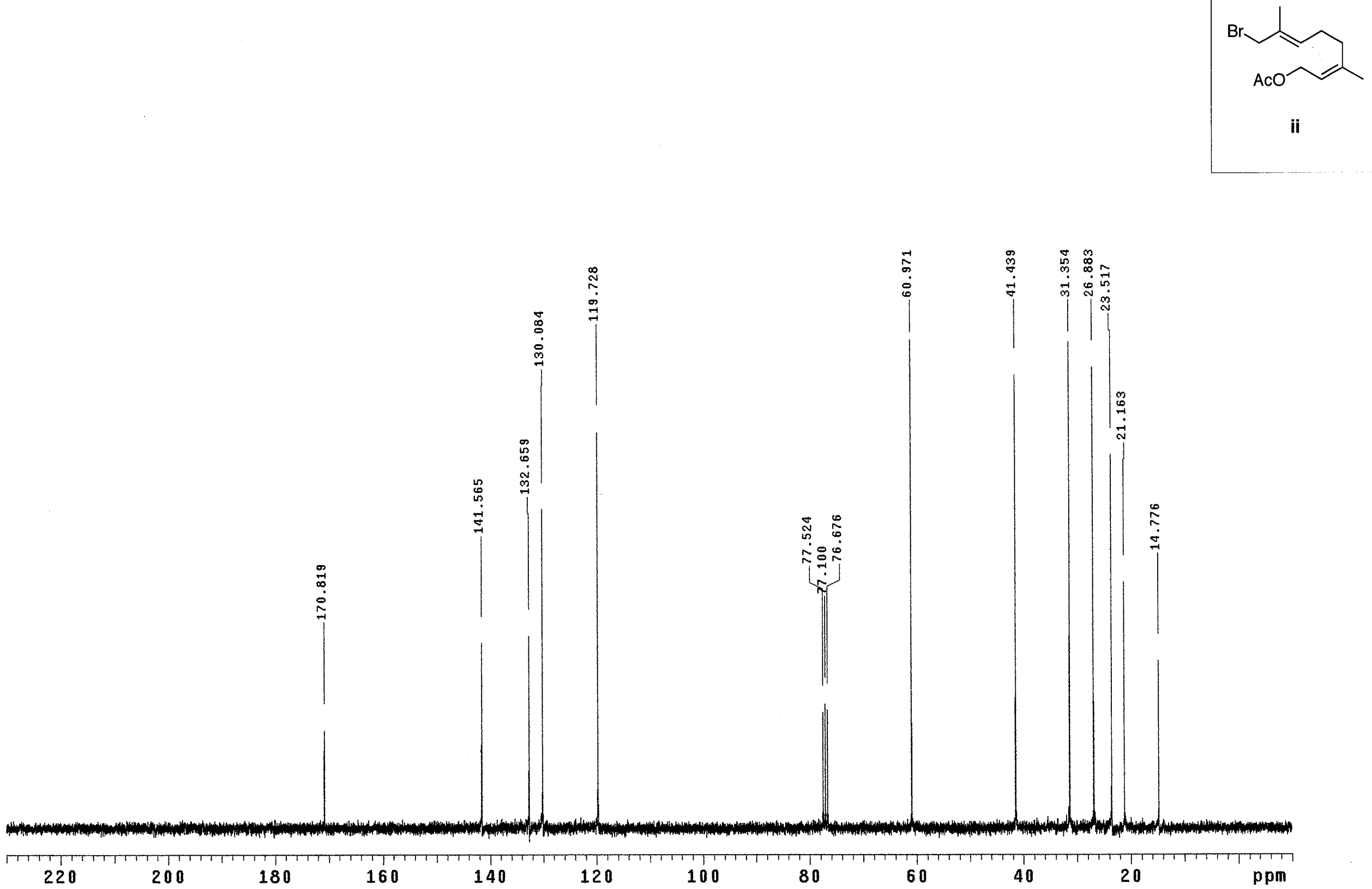

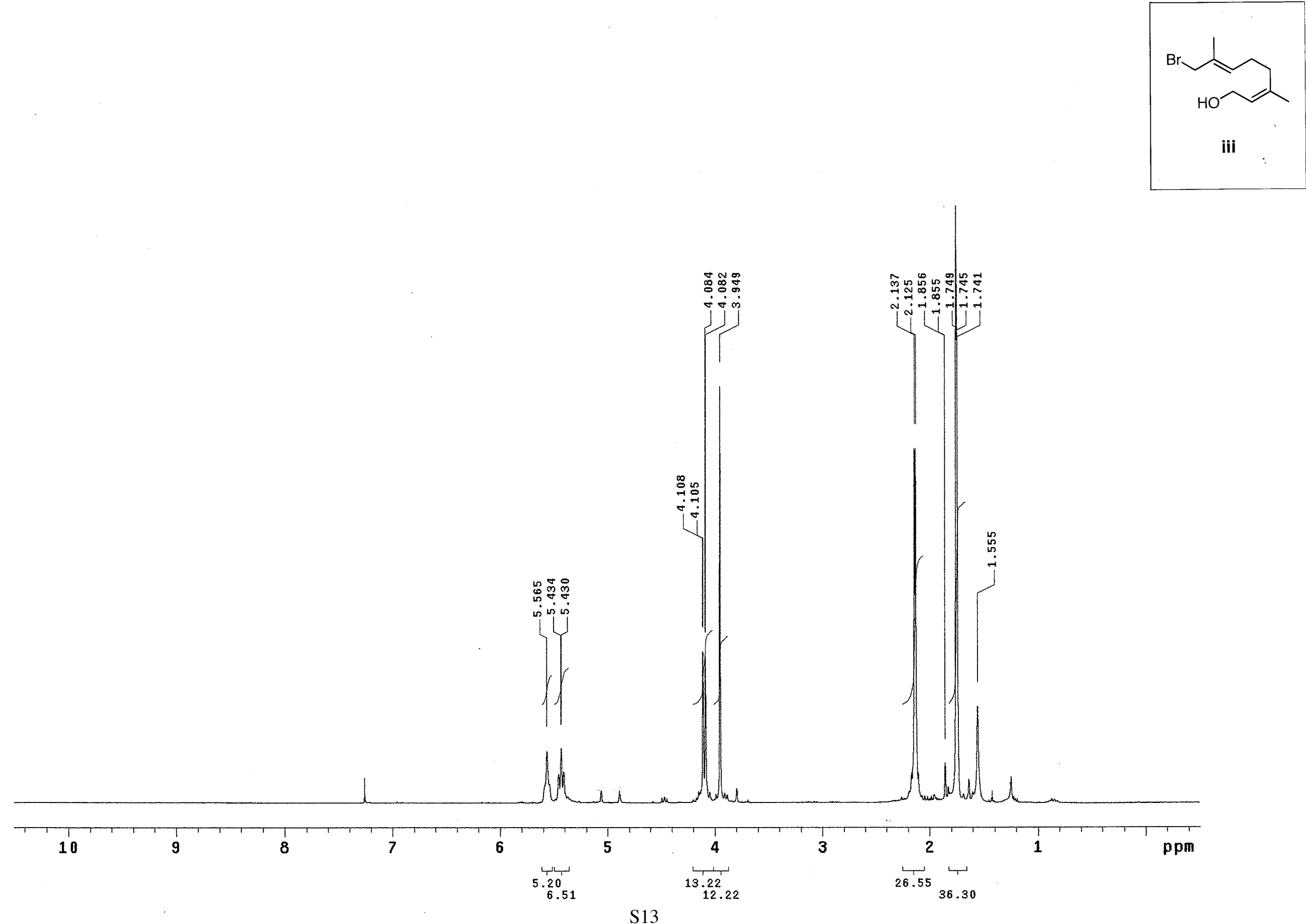

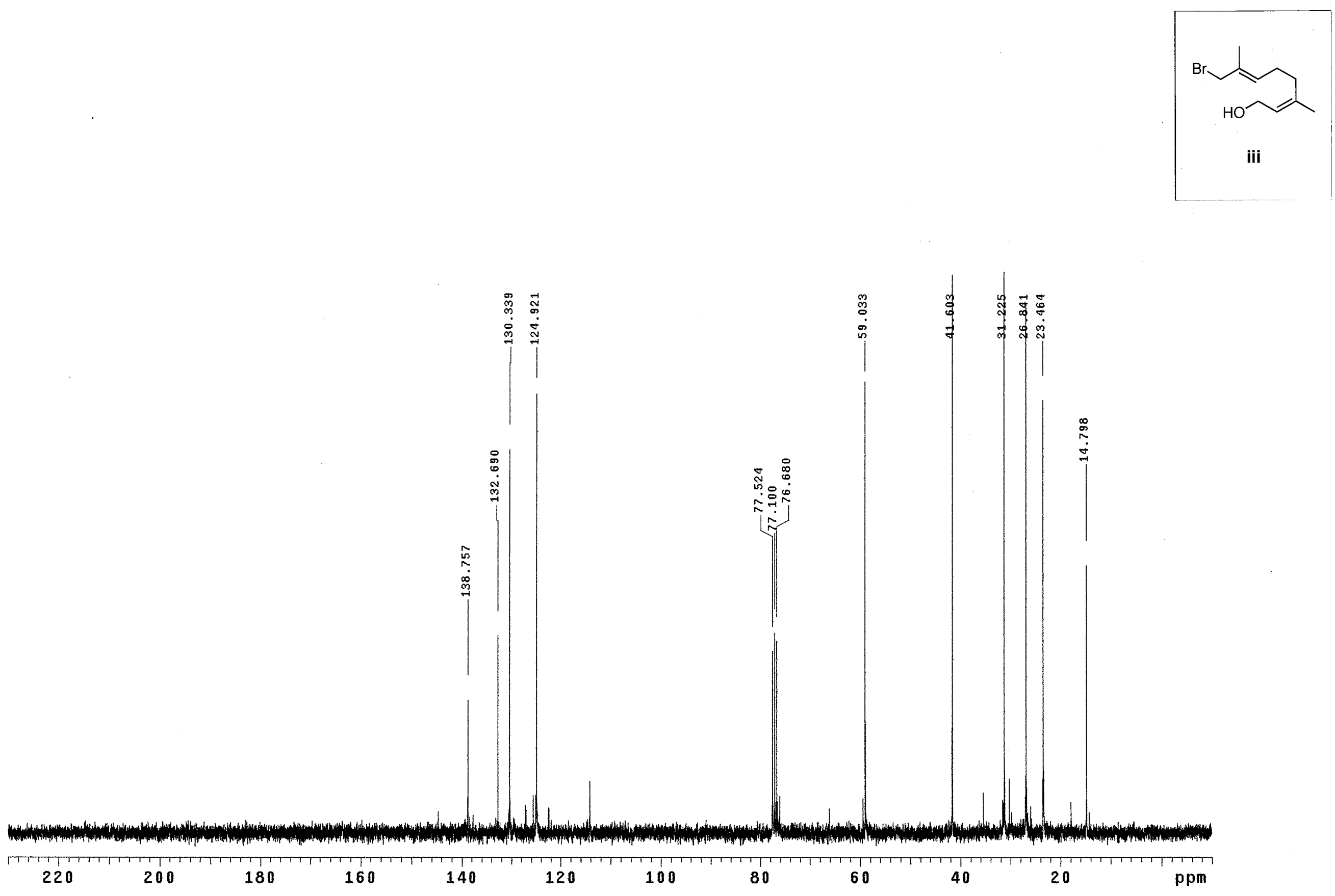

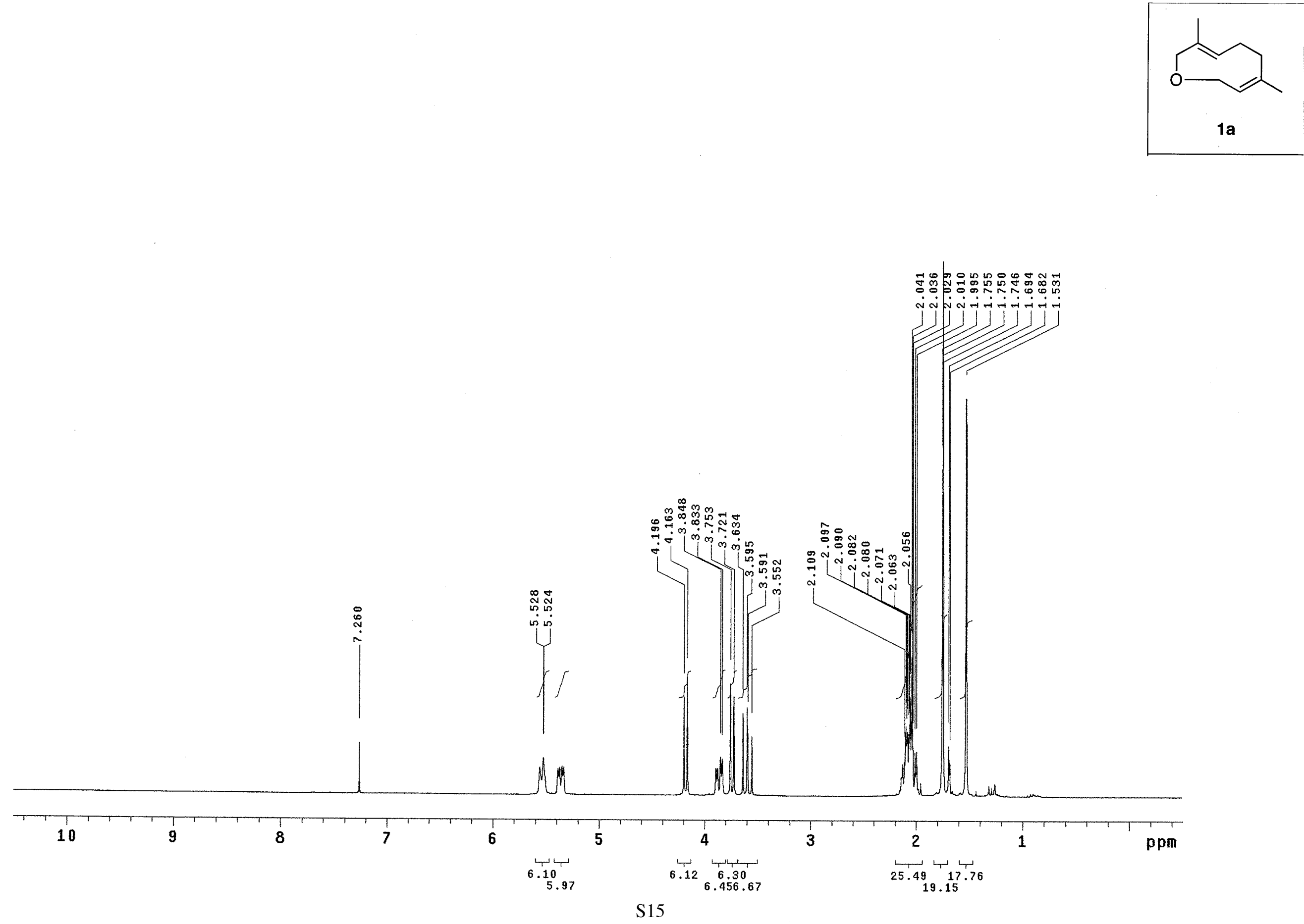

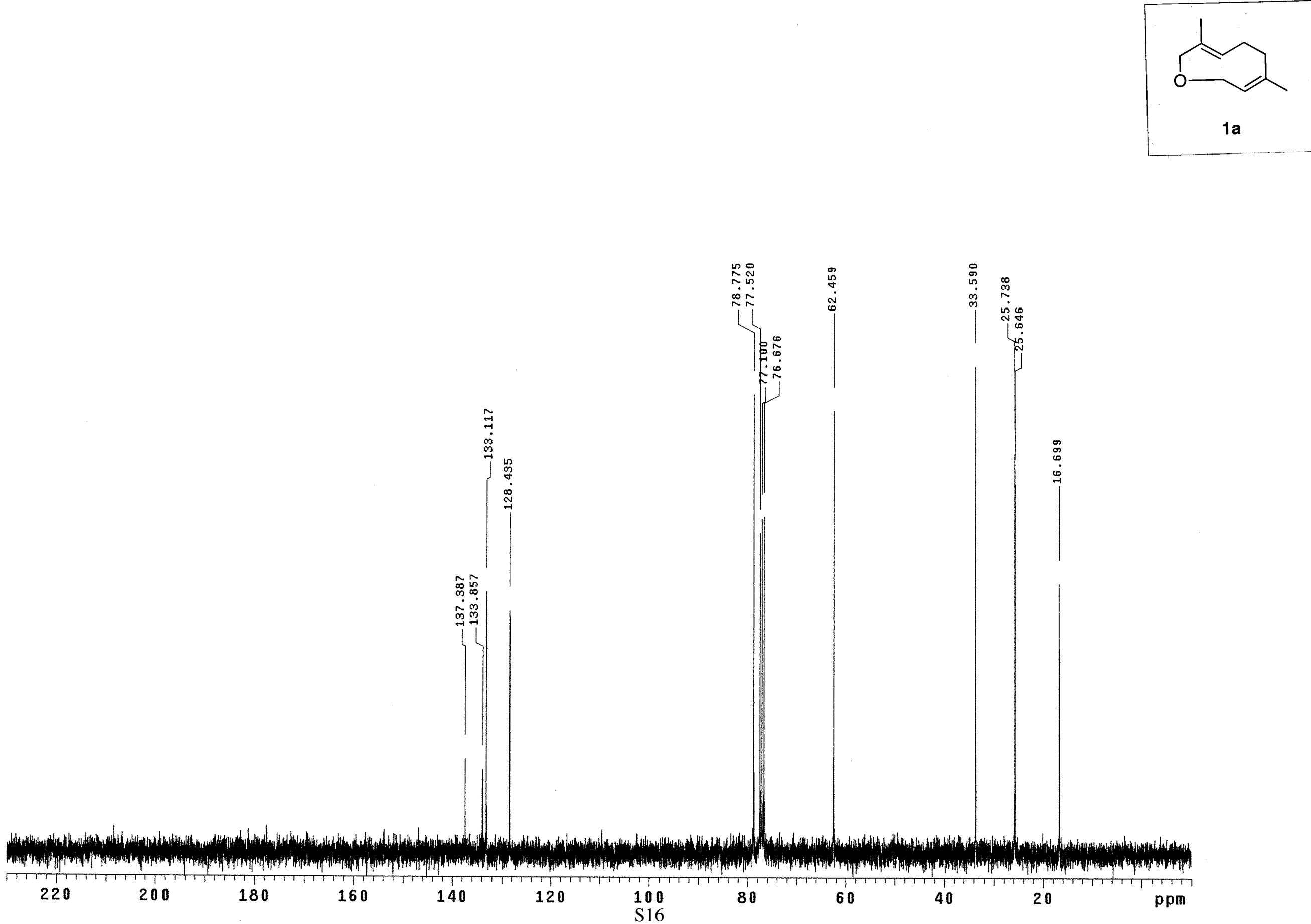
HPLC analysis (CD and UV)

CHIRALCEL OD-H (4.6 X 250 mm)

hexane $/ 2$-propanol $=500: 1,0.3 \mathrm{~mL} / \mathrm{min}$

UV $220 \mathrm{~nm}$

$1.0 \mathrm{E}+04 \mid \mu \mathrm{V}$

$8.0 \mathrm{E}+03$

$1 a$

$6.0 \mathrm{E}+03$

$4.0 \mathrm{E}+03$

$2.0 \mathrm{E}+03$

$0.0 \mathrm{E}+00$

$-2.0 \mathrm{E}+03$

$-4.0 \mathrm{E}+03$

$-6.0 \mathrm{E}+03$

$-8.0 \mathrm{E}+03$

$2.0 \mathrm{E}+05 \mu \mathrm{V}$

5.00

10.00

15.00

20.00

25.00

$30.00[\mathrm{~min}]$

$1.5 \mathrm{E}+05$

$1.0 \mathrm{E}+05$

$5.0 \mathrm{E}+04$

$0.0 E+00$

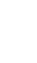

(1)

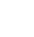

00

ファイル名: KU558-003. CH1

コメント:

Vial \# = 1 Rack \# = 1

注入日：2-Jul-2004 15:37:00

現在日時: 2-Jul-2004 16:47:12

ユーザー： UEHARA

グループ： TOMOOKA

システムプログラム:HEX500-IPA1-UV220

\# ピーク名 Rt 面積 $[\mu \mathrm{V}$. S Sec $] \quad$ 定量值 面積\%

$\begin{array}{lllll}1 & 21.742 & 381256.869 & 0.000 & 49.48 \\ 2 & 24.067 & 389259.779 & 0.000 & 50.52\end{array}$

ピーク総面積 $=770516.647$ [ $\mu \mathrm{V} . \mathrm{Sec}]$

ファイル名: KU558-003. CH2

コメント:

Vial \#= 1 Rack \# = 1

注入日：2-Jul-2004 15:37:00

現在日時: 2-Jul-2004 16:47:12

ユニー゙ニ： UEHARA

グループ：TOMOOKA

システムプログラム:HEX500-IPA1-UV220

\# ピーク名 Rt 面積 $[\mu \mathrm{V} . \mathrm{Sec}] \quad$ 定量値 面積\%

$\begin{array}{lllll}1 & 21.758 & 4765359.417 & 0.000 & 50.26 \\ 2 & 24.067 & 4716589.000 & 0.000 & 49.74\end{array}$

ピーク総面積 $=9481948.417$ [ $\mu$ V. Sec] 


\section{CD and UV analysis}

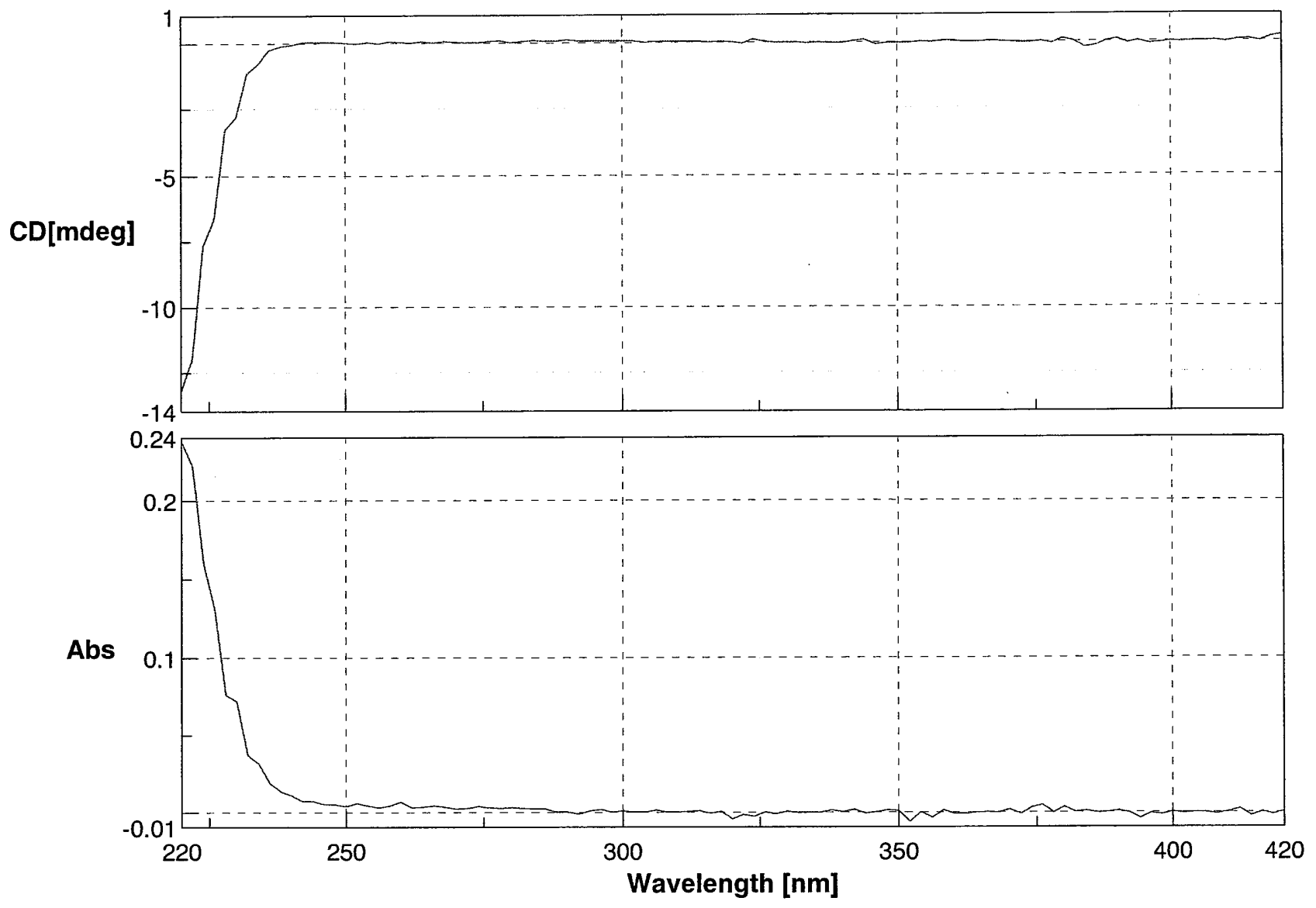

旦時

機種名

シリアアル番号

試料名

測定者
2004/06/30 19:59

KU558-1. jws

CD-2095

003460875

$C D / U V$ Spectra $t R=0.000 \mathrm{~min}$

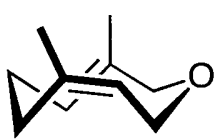

$(-)-(S)-1 a$ 
CD and UV analysis

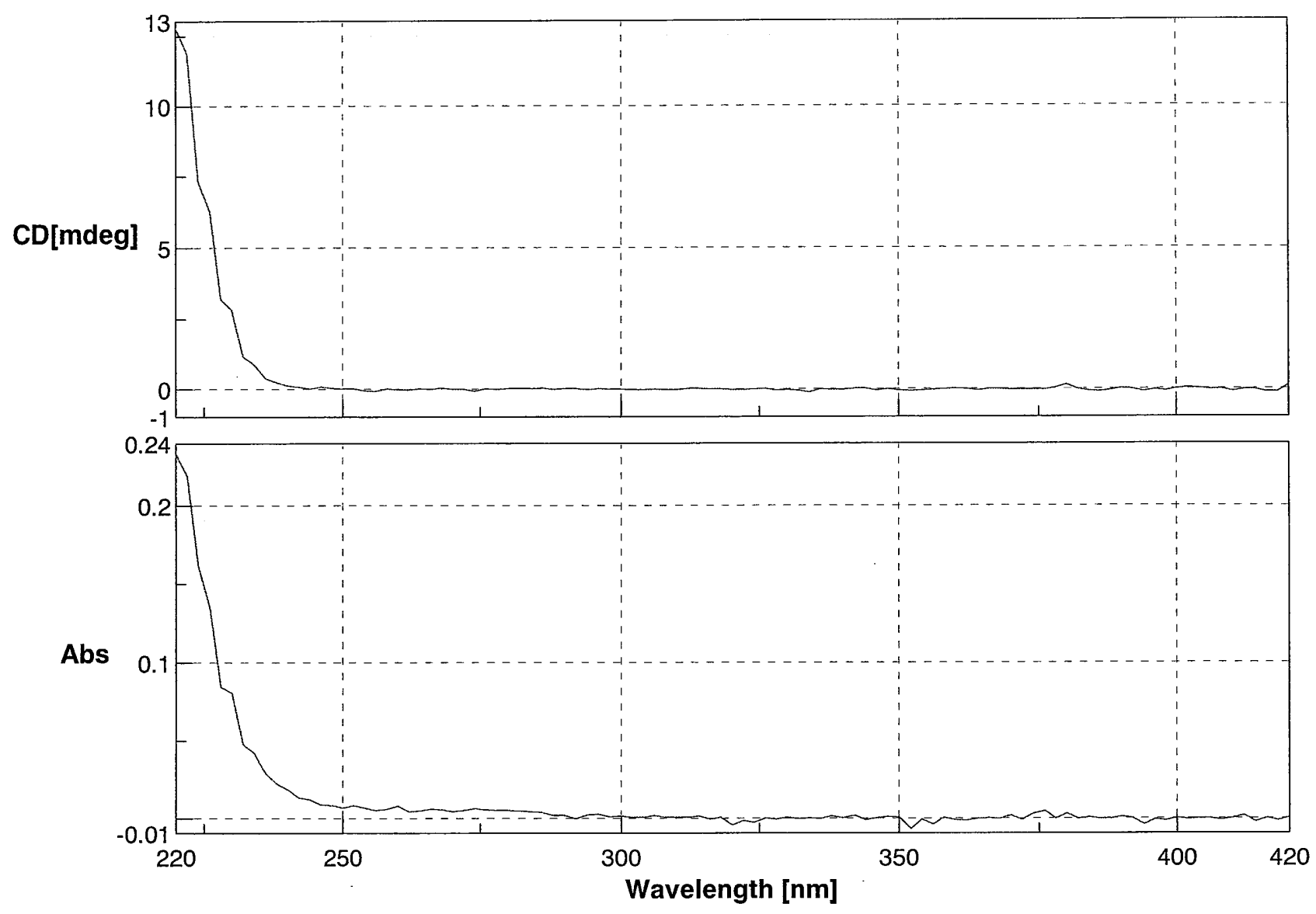

日時

フアイル名

機種名

シリ予ル番号

2004/06/30 20:01

KU558-2. jws

CD-2095

003460875

試料名

測定者

コメシト

$C D / U V$ Spectra $t R=0.000 \mathrm{~min}$

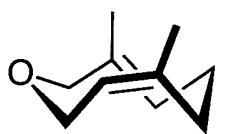

$(+)-(R)-1 \mathrm{a}$ 


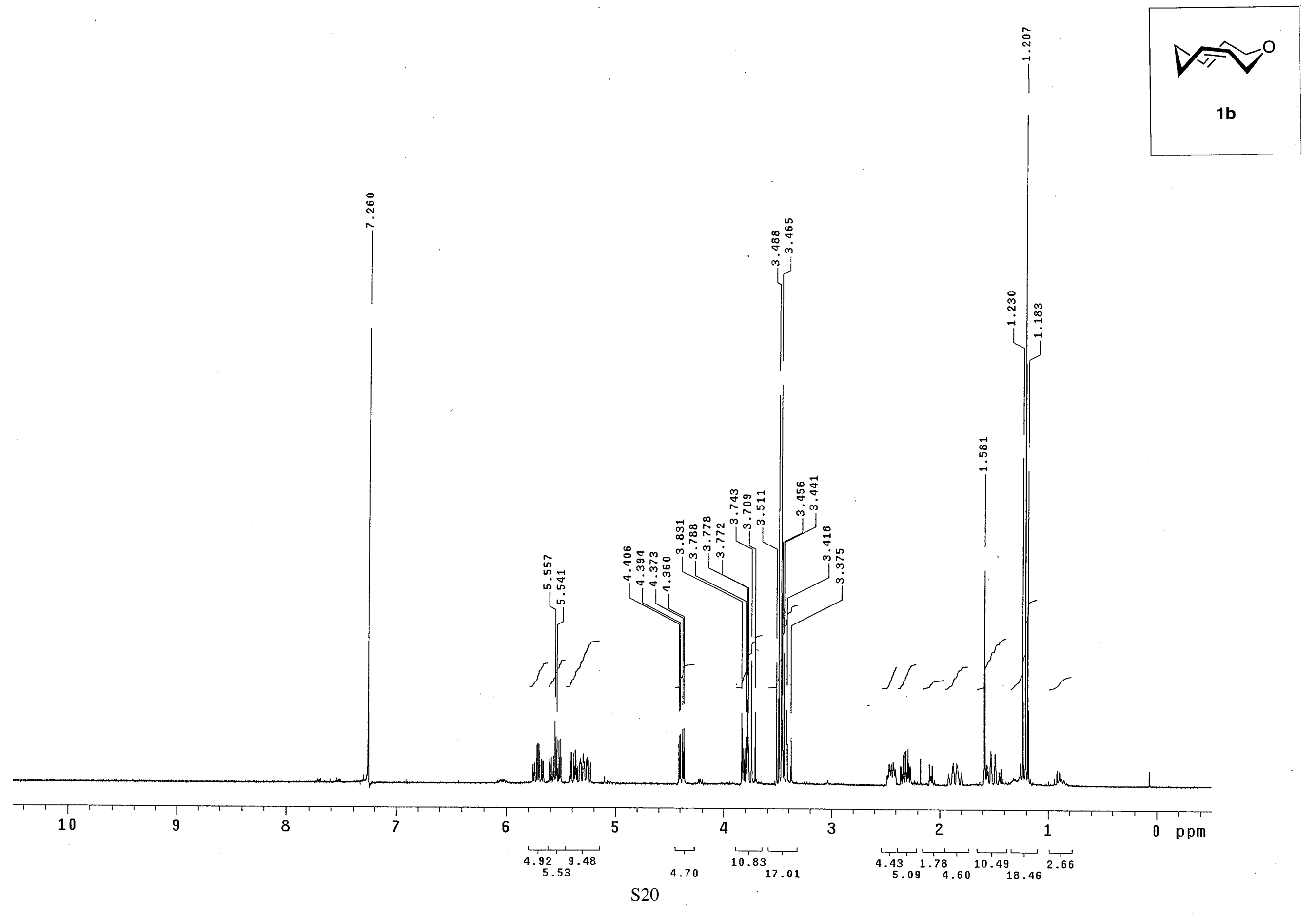



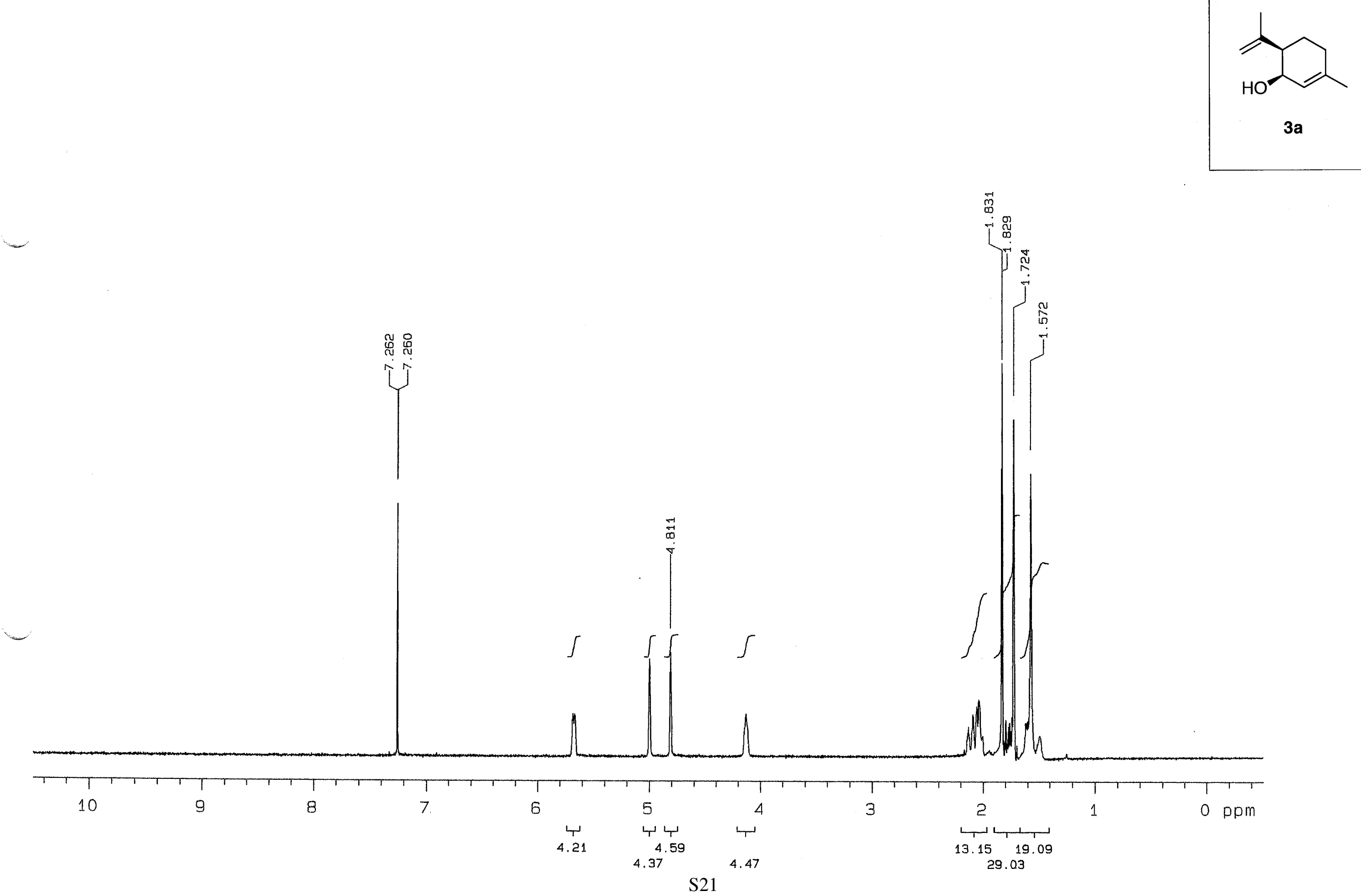


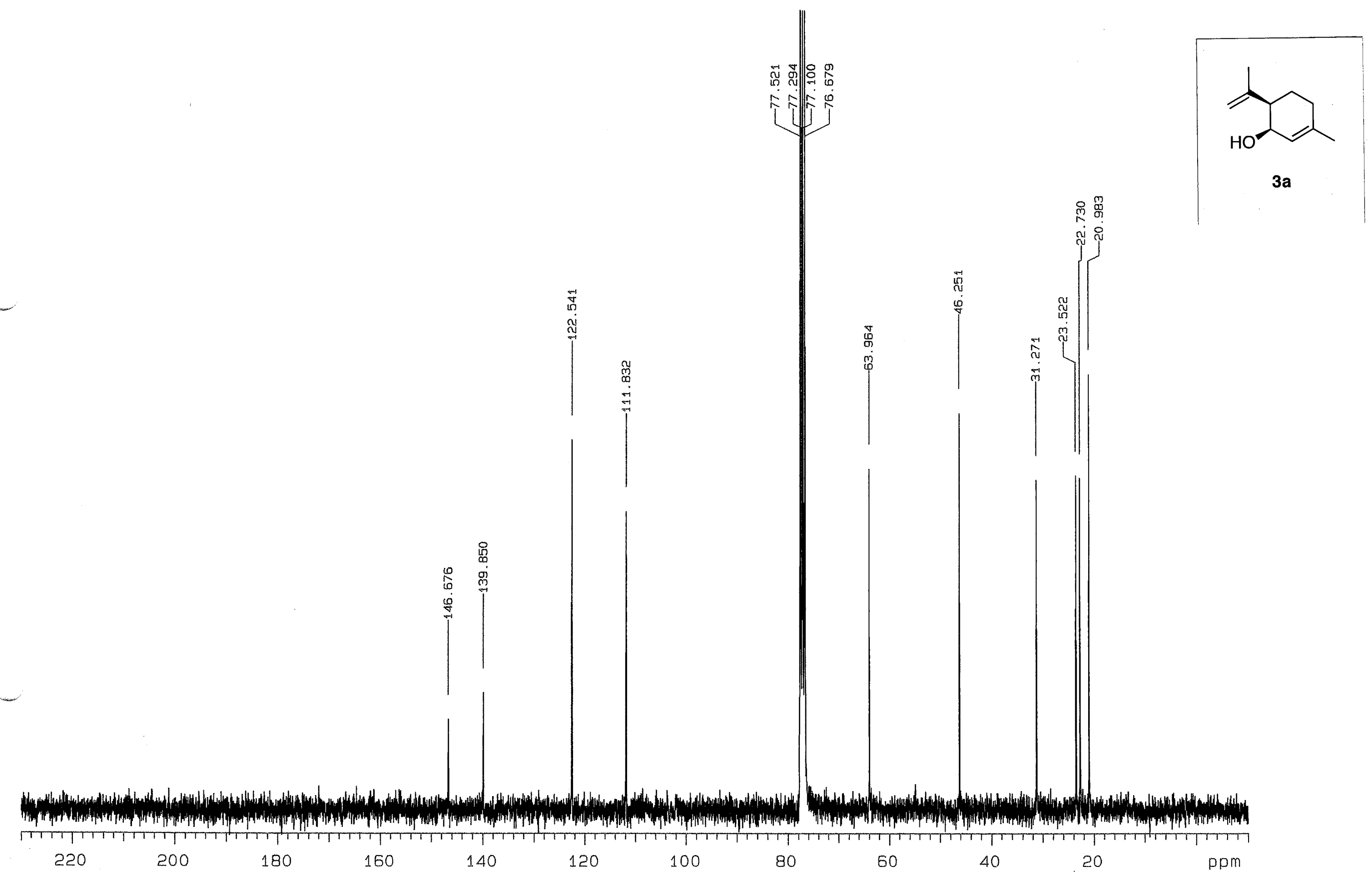




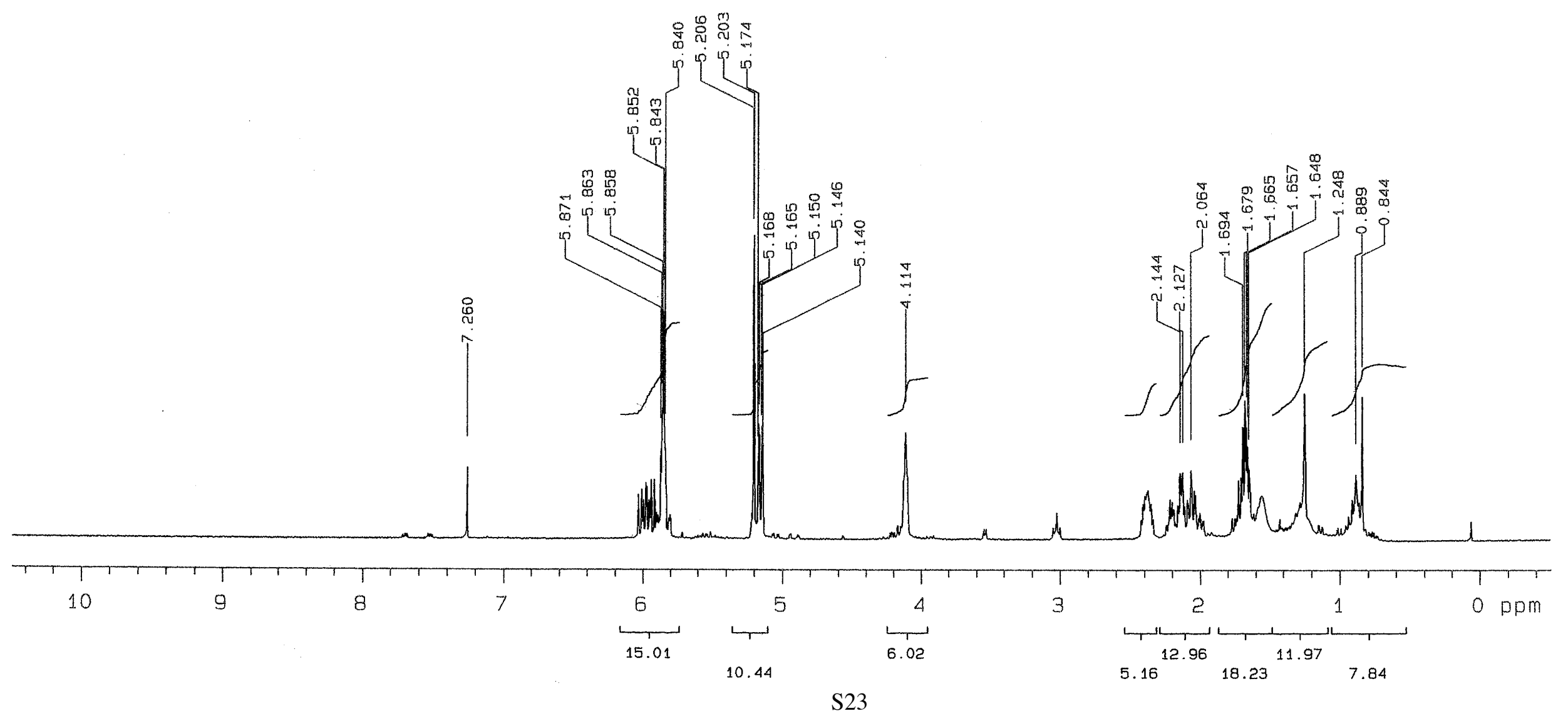




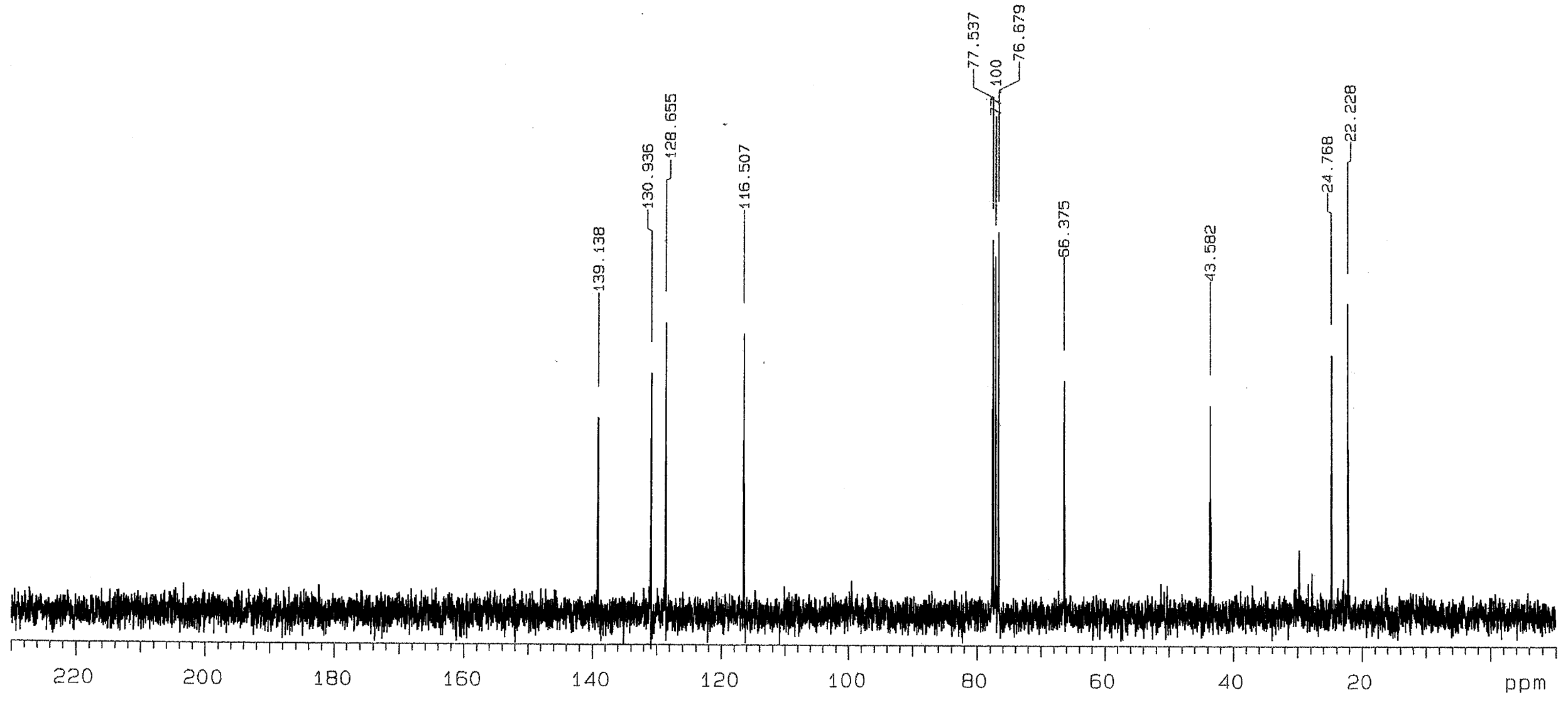




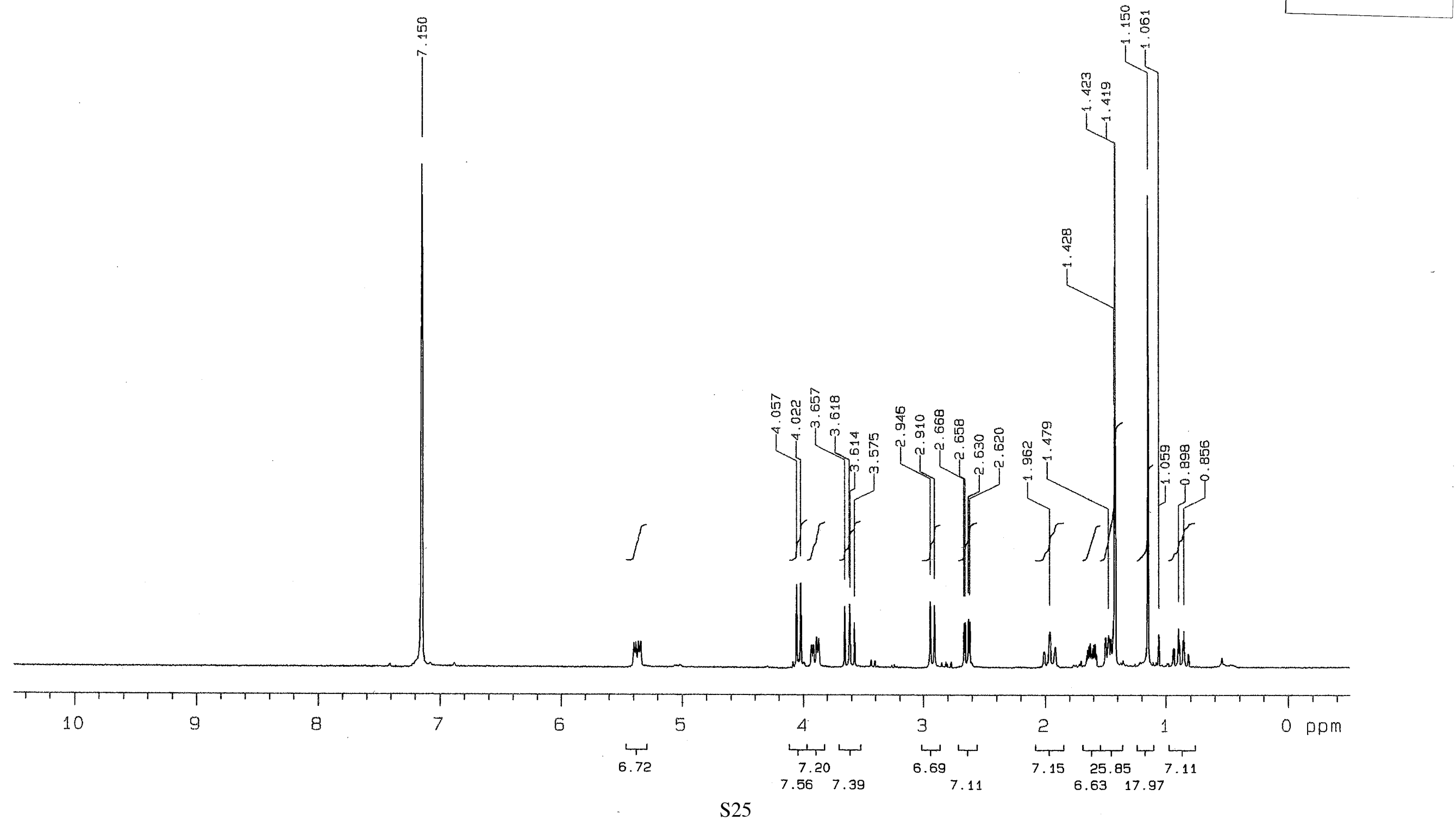




$$
1
$$




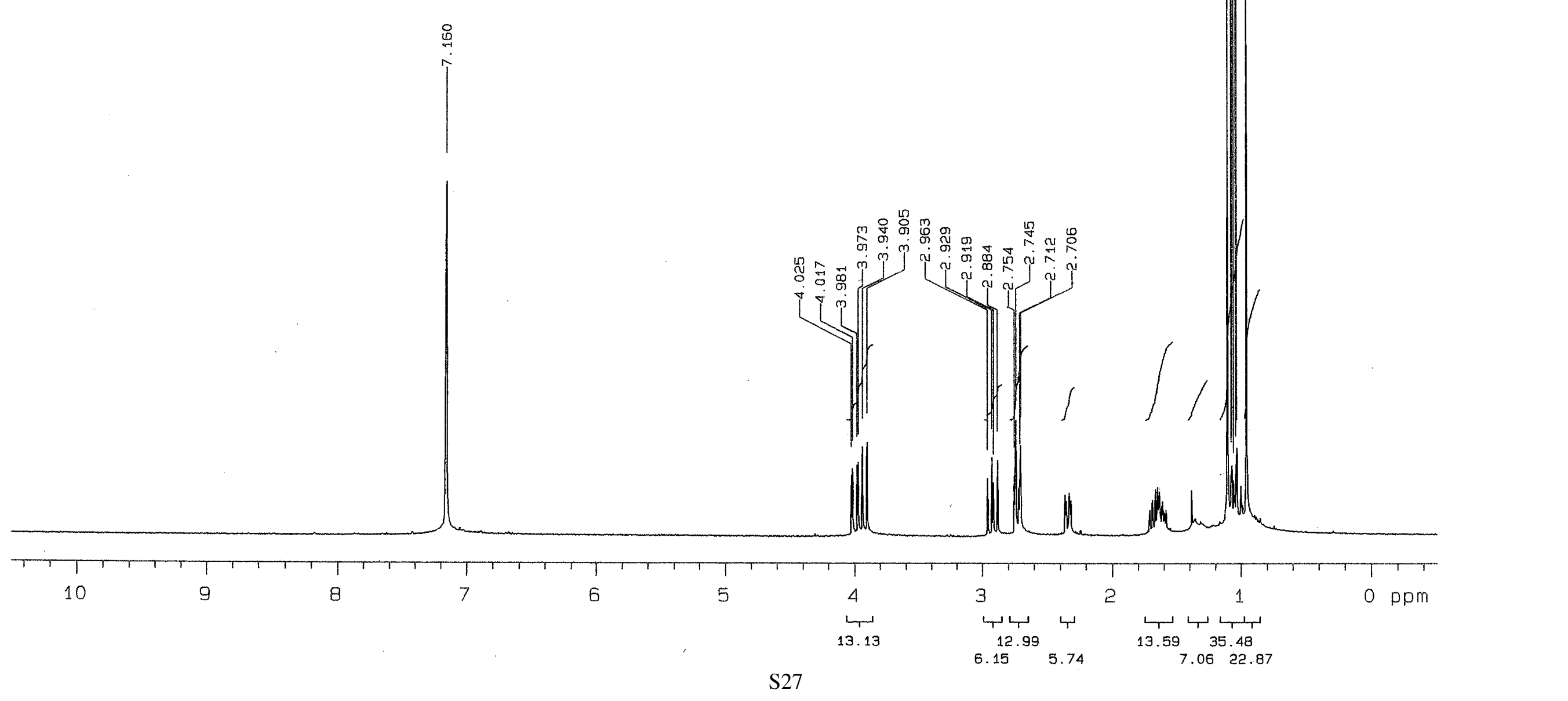




$$
11
$$




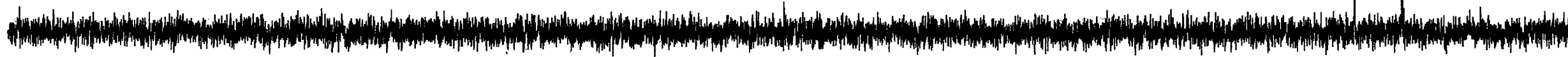

CH2 carbons

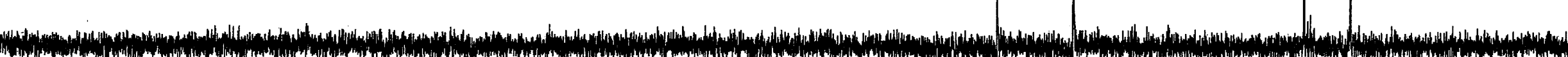
H

$\mathrm{CH}$ carbons

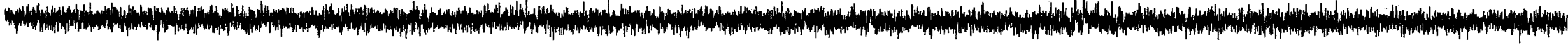
v

all protonated carbons

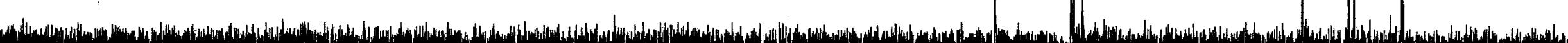

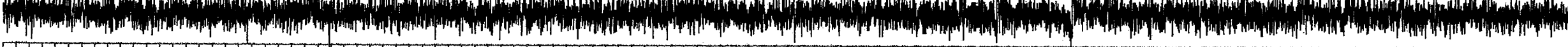

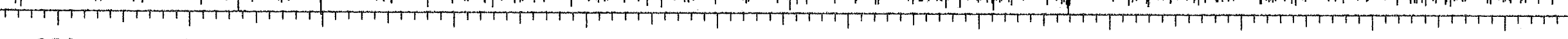



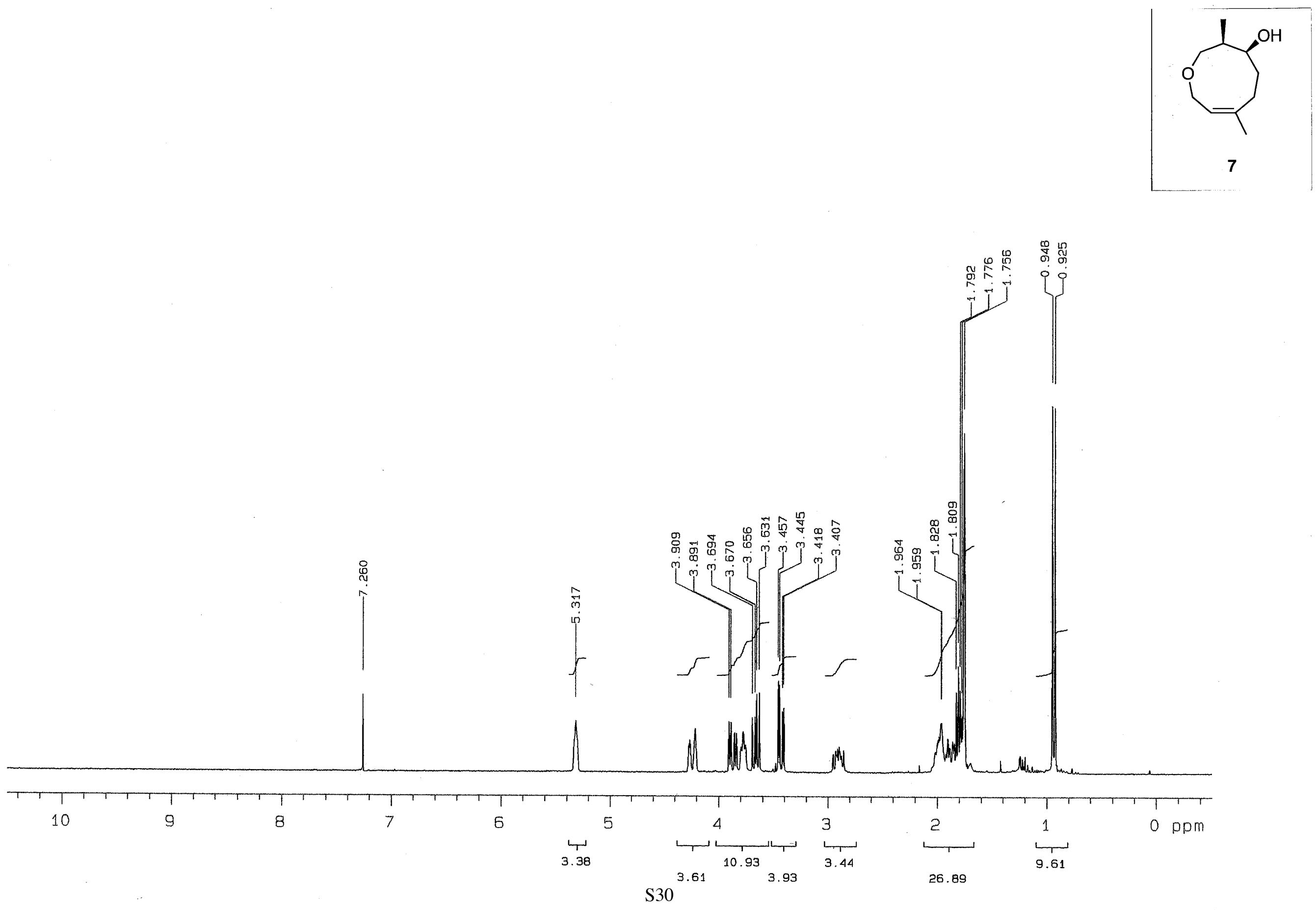

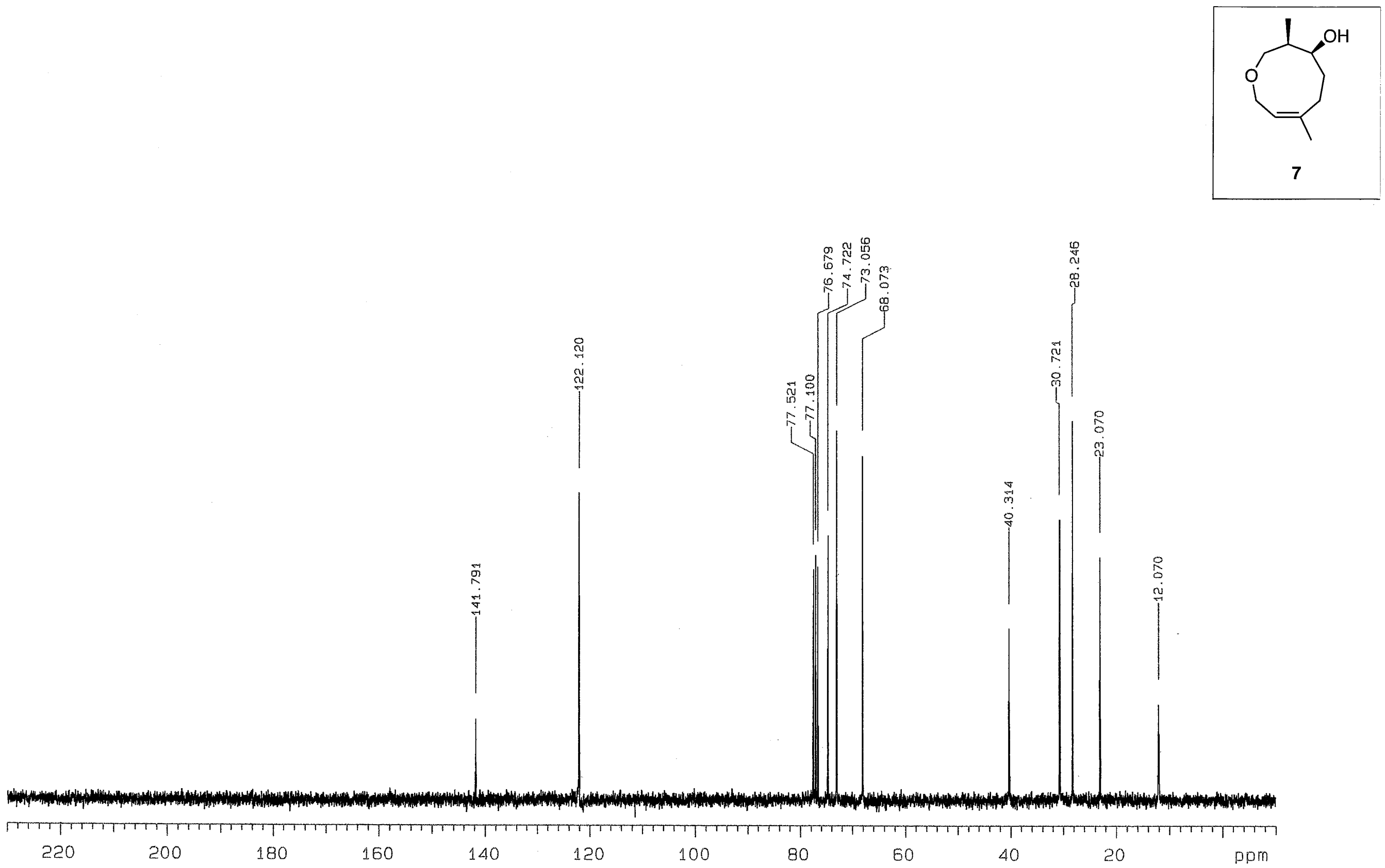


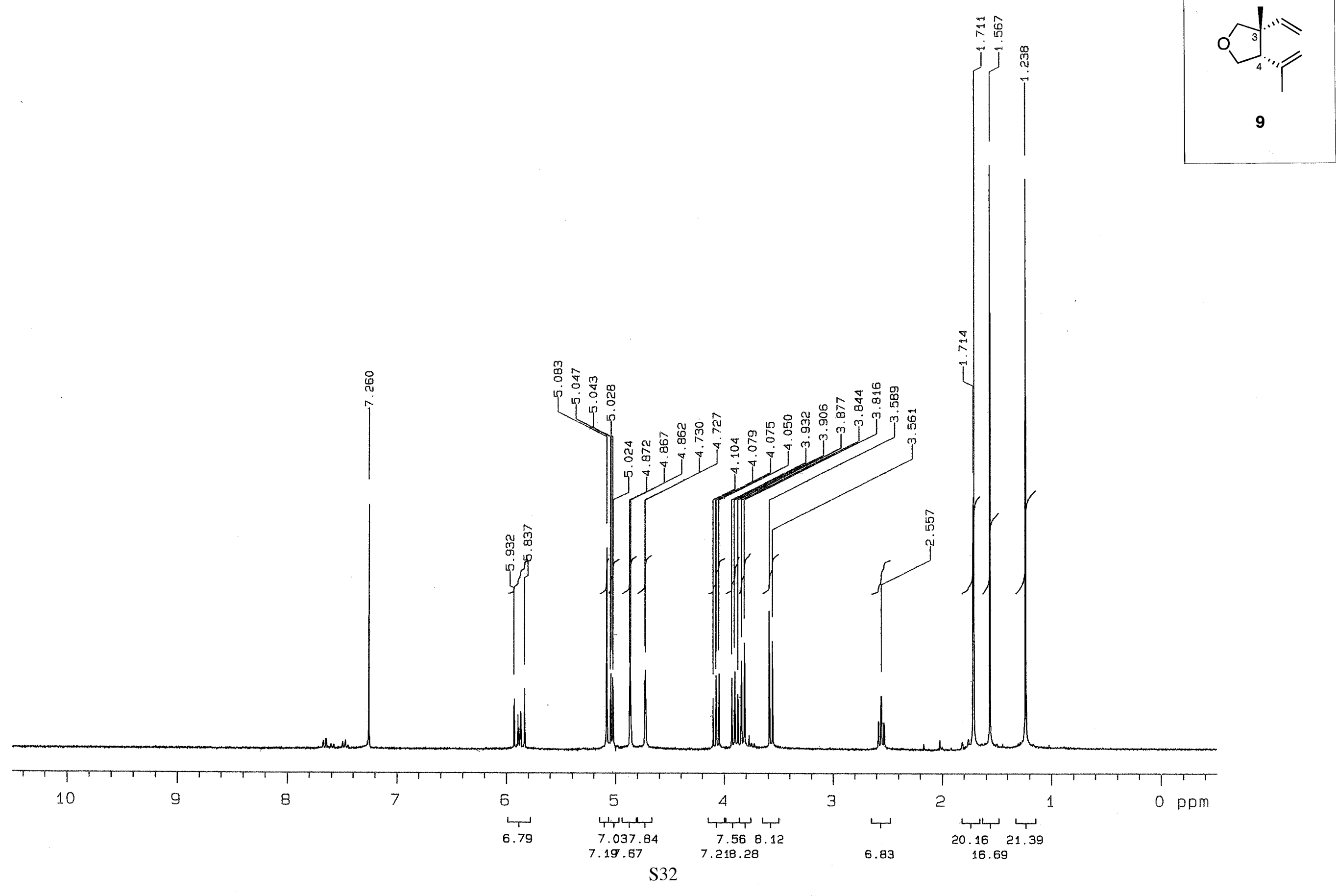




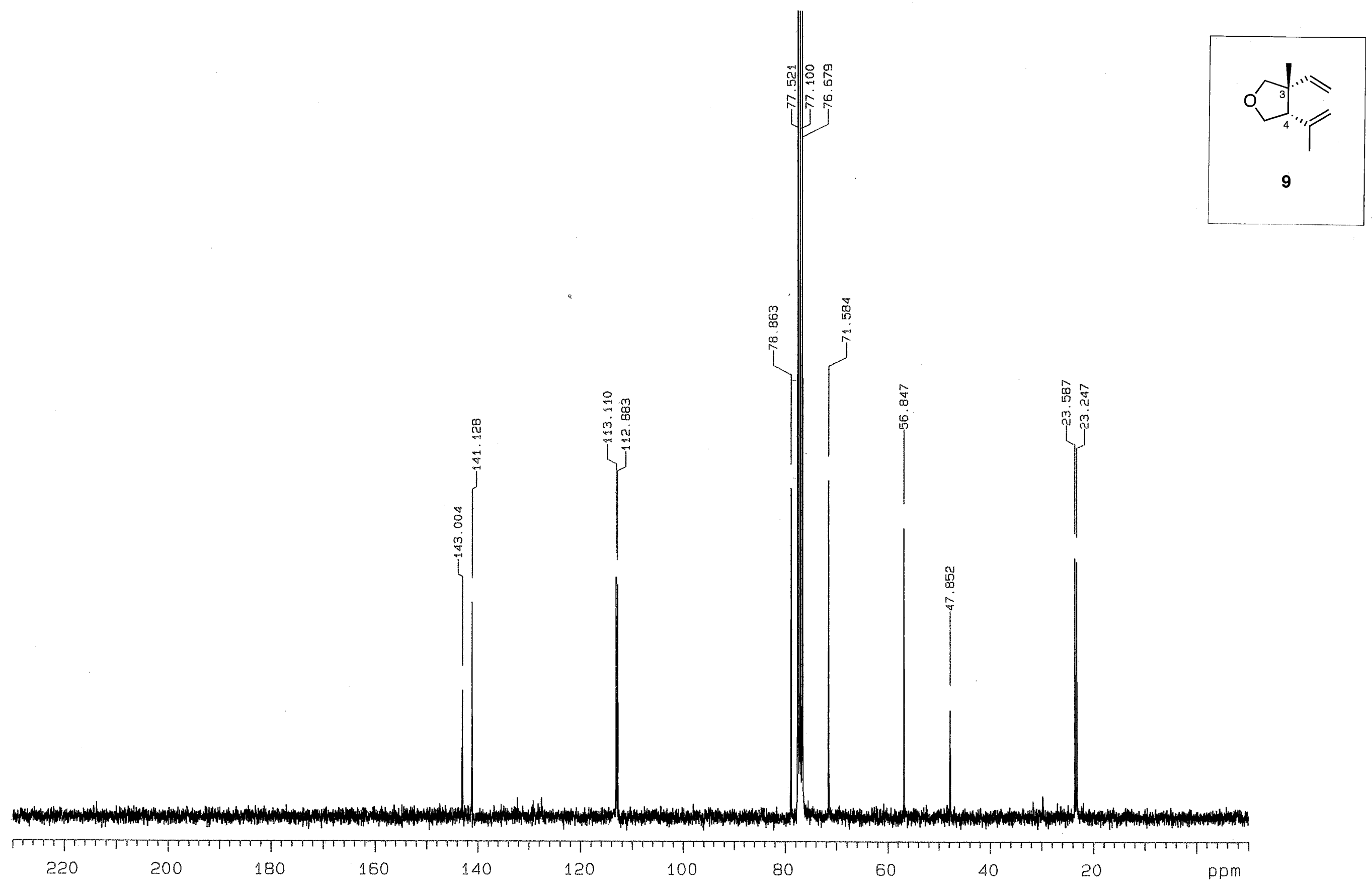

